\title{
New Candidate Extreme T Subdwarfs from the Backyard Worlds: Planet 9 Citizen Science Project
}

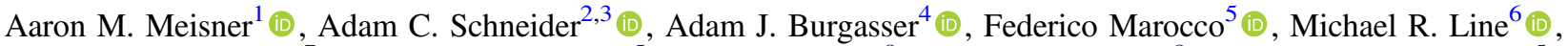
Jacqueline K. Faherty $^{7}$ (1) J. Davy Kirkpatrick ${ }^{5}$ (1) , Dan Caselden $^{8}$ (1) , Marc J. Kuchner ${ }^{9}$ (1), Christopher R. Gelino ${ }^{5}$, Jonathan Gagné ${ }^{10,11}$ (1) , Christopher Theissen ${ }^{4,19}$ (1) , Roman Gerasimov ${ }^{4}$ (1) , Christian Aganze ${ }^{4}$ (), Chih-chun Hsu ${ }^{4}$ (1), John P. Wisniewski ${ }^{12}$ (10), Sarah L. Casewell ${ }^{13}$ (1), Daniella C. Bardalez Gagliuffi ${ }^{7}$ (1), Sarah E. Logsdon ${ }^{1}$ (1) Peter R. M. Eisenhardt $^{14}$, Katelyn Allers ${ }^{15}$ (D), John H. Debes ${ }^{16}$ (1), Michaela B. Allen ${ }^{9}$, Nikolaj Stevnbak Andersen ${ }^{17}$ (1), Sam Goodman ${ }^{17}$ (1), Léopold Gramaize ${ }^{17}$ (1), David W. Martin ${ }^{17}$, Arttu Sainio ${ }^{17}$ (1) and Michael C. Cushing ${ }^{18}$ (1) The Backyard Worlds: Planet 9 Collaboration

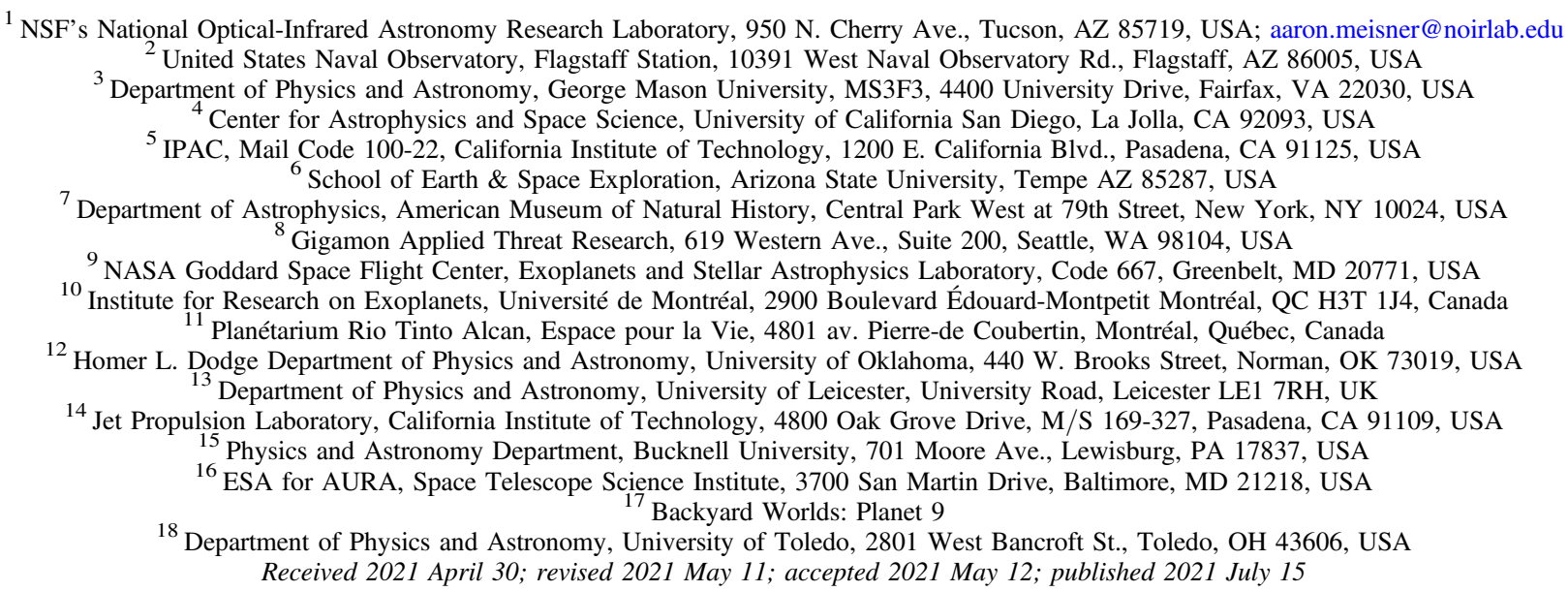

\begin{abstract}
Schneider et al. presented the discovery of WISEA J041451.67-585456.7 and WISEA J181006.18-101000.5, which appear to be the first examples of extreme T-type subdwarfs (esdTs; metallicity $\leqslant-1$ dex, $T_{\text {eff }} \lesssim 1400 \mathrm{~K}$ ). Here, we present new discoveries and follow-up of three T-type subdwarf candidates, with an eye toward expanding the sample of such objects with very low metallicity and extraordinarily high kinematics, properties that suggest membership in the Galactic halo. Keck/NIRES near-infrared spectroscopy of WISEA J155349.96 +693355.2, a fast-moving object discovered by the Backyard Worlds: Planet 9 citizen science project, confirms that it is a mid-T subdwarf. With $H_{W 2}=22.3$ mag, WISEA J155349.96+693355.2 has the largest W2 reduced proper motion among all spectroscopically confirmed L and T subdwarfs, suggesting that it may be kinematically extreme. Nevertheless, our modeling of the WISEA J155349.96+693355.2 near-infrared spectrum indicates that its metallicity is only mildly subsolar. In analyzing the J155349.96+693355.2 spectrum, we present a new grid of low-temperature, low-metallicity model atmosphere spectra. We also present the discoveries of two new esdT candidates, CWISE J073844.52-664334.6 and CWISE J221706.28-145437.6, based on their large motions and colors similar to those of the two known esdT objects. Finding more esdT examples is a critical step toward mapping out the spectral sequence and observational properties of this newly identified population.
\end{abstract}

Unified Astronomy Thesaurus concepts: Brown dwarfs (185); T dwarfs (1679); T subdwarfs (1680)

\section{Introduction}

What are the physical properties of the lowest-luminosity substellar objects in our the Milky Way? How do brown dwarf atmospheres and spectral energy distributions change as a function of multiple variables, including temperature, metallicity, and age? How has the birth rate of substellar objects evolved over time, from early periods of star formation in the universe to the present epoch? Ultracool subdwarfs, very lowmass stars and brown dwarfs with low metallicity that can be found in the Milky Way thick disk or halo, are important

\footnotetext{
${ }^{19}$ NASA Sagan Fellow.
}

laboratories that play a major role in answering these questions (e.g., Burgasser et al. 2005).

The solar neighborhood provides our best opportunity to study the Galactic substellar population across its span of temperatures, metallicities, ages, and masses. Pinpointing nearby substellar objects representing the extremes of these parameters is therefore a key way to test/develop theoretical models, such as those of giant exoplanet atmospheres (e.g., Reid \& Metchev 2008; Marley \& Robinson 2015; Leggett et al. 2019) and the star formation process at low masses (e.g., Kirkpatrick et al. 2019a, 2021).

Hundreds of $\mathrm{T}$ dwarfs ( $450 \mathrm{~K} \lesssim \mathrm{T}_{\text {eff }} \lesssim 1400 \mathrm{~K}$; e.g., Nakajima et al. 1995; Kirkpatrick et al. 2011; Mace et al. 2013a) and dozens of low-metallicity T subdwarfs (e.g., Burningham et al. 2014; 
Pinfield et al. 2014; Zhang et al. 2019) are now known. Recently, the Backyard Worlds: Planet 9 citizen science project (backyardworlds.org; Kuchner et al. 2017, henceforth Backyard Worlds) discovered the first two examples of "extreme T-type subdwarfs" (esdTs; Schneider et al. 2020), with $T_{\text {eff }} \lesssim 1400 \mathrm{~K}$ and $[\mathrm{Fe} / \mathrm{H}] \leqslant-1$. The esdTs have distinctive near-infrared (NIR) colors unlike those of other brown dwarfs and spectra not wellreplicated by any existing models. Determining the space densities of very cold and metal-poor objects, such as esdTs, may yield new insights about the dependence of low-mass star formation on metallicity throughout cosmic time. The low-metallicity atmospheres of the coldest isolated subdwarfs will also help us understand (by proxy) the atmospheres of giant exoplanets that orbit old and/or metal-poor host stars.

To further map out the parameter space of $\mathrm{T}$ subdwarfs with very low metallicity and extreme kinematics, we searched through thousands of Backyard Worlds moving object discoveries, identifying new esdT candidates based on peculiar infrared colors and large proper motion. This yielded three new extreme $\mathrm{T}$ subdwarf candidates: CWISE J073844.52-664334.6 (hereafter CWISE 0738-6643), WISEA J155349.96+693355.2 (Meisner et al. 2020, hereafter WISEA 1553+6933) and CWISE J221706.28-145437.6 (hereafter CWISE 2217-1454).

In Section 2, we provide a brief overview of the Backyard Worlds citizen science project. In Section 3, we describe our selection of new esdT candidates from within the full list of Backyard Worlds moving object discoveries. In Section 4, we introduce the three esdT candidates identified. In Section 5, we present Keck/NIRES spectroscopy of WISEA 1553+6933, including comparison against an extensive new grid of lowtemperature, low-metallicity model atmosphere spectra. In Section 6, we investigate the synthetic colors of our new models as a function of metallicity. We conclude in Section 7.

\section{The Backyard Worlds: Planet 9 Citizen Science Project}

Backyard Worlds (Kuchner et al. 2017) is a moving object search that crowdsources the visual inspection of all-sky Widefield Infrared Survey Explorer (WISE; Wright et al. 2010) images among thousands of online volunteers. The project launched in 2017 February via the Zooniverse web platform (Simpson et al. 2014). Volunteers scrutinize time series image blinks (referred to as "flipbooks"), with each flipbook showing the evolution of a random $10^{\prime} \times 10^{\prime}$ sky patch over the 2010-2016 time period. Each flipbook frame is a two-color composite representing one WISE sky pass, with WISE W1 $(3.4 \mu \mathrm{m})$ encoded as the blue channel and W2 $(4.6 \mu \mathrm{m})$ as the red channel. Backyard Worlds pushes fainter than prior WISE-based motion searches because its flipbooks are built from deep/clean time-resolved "unWISE" coadds (Lang 2014; Meisner et al. 2018) that offer a long 6 yr time baseline. The signature Backyard Worlds science application is discovering cold brown dwarfs in the solar neighborhood based on their characteristic combination of high proper motion and red W1-W2 color. Backyard Worlds discoveries have spanned multiple subtopics within solar neighborhood science, including extremely cold Y dwarfs (Bardalez Gagliuffi et al. 2020; Meisner et al. 2020), co-moving companions (e.g., Faherty et al. 2020; Jalowiczor et al. 2021; Rothermich et al. 2021), T dwarfs (e.g., Kuchner et al. 2017), white dwarf disks (Debes et al. 2019), and previously overlooked members of the 20 pc brown dwarf census (Kirkpatrick et al. 2021). Backyard Worlds revealed the first two known examples of extreme T subdwarfs (Schneider et al. 2020).
Backyard Worlds motion searches have expanded significantly beyond the project's Zooniverse interface. For instance, Backyard Worlds citizen scientists have created novel visualization tools to interactively customize WISE image blinks (e.g., WiseView; Caselden et al. 2018). A group of $\sim 300$ advanced users has conducted numerous catalog-based brown dwarf candidate queries on platforms like IRSA and Astro Data Lab (Fitzpatrick et al. 2019), particularly leveraging the unWISE Catalog (Schlafly et al. 2019) and CatWISE 2020 (Marocco et al. 2021), the latter offering WISE-based proper motion measurements $>10 \times$ more accurate than those of AllWISE (Cutri et al. 2013). Because Backyard Worlds participants scan WISE images by eye for any/all moving sources, the project is well-positioned to discover atypical objects like subdwarfs that might be missed by traditional color searches (e.g., Griffith et al. 2012).

\section{Selection of New Extreme T Subdwarf Candidates}

As of 2021 April, Backyard Worlds participants have discovered roughly 3200 motion-confirmed L, T, and Y dwarf candidates. Most of these discoveries are likely to be "ordinary" L and T dwarfs. We mined this large list of Backyard Worlds discoveries to determine whether any may be promising new candidate members of the esdT spectral class. Typically, very little information about our Backyard Worlds motion discoveries is available, since the sample is generally quite faint and red by selection. In many cases, only $\mathrm{W} 1$ and W2 detections are available from archival survey data sets.

Aside from $\mathrm{W} 1$ and $\mathrm{W} 2, \mathrm{~J}$ band is the most valuable filter at our disposal for which sensitive archival survey photometry exists over most of the sky. We therefore sought to identify esdT candidates based on the combination of $\mathrm{W} 1, \mathrm{~W} 2$, and $\mathbf{J}$ photometry. esdTs inhabit a distinctive region of $\mathrm{J}-\mathrm{W} 2$ versus W1-W2 color-color space (see Figure 1, which is based on Figure 3 of Schneider et al. 2020). esdTs have much redder $\mathbf{J}$ $-\mathrm{W} 2$ colors than do $\mathrm{T}$ dwarfs in the same $1.1 \mathrm{mag} \leqslant \mathrm{W} 1$ $-\mathrm{W} 2 \leqslant 1.75$ mag WISE color range.

For all 3200 Backyard Worlds moving object discoveries, we compiled available 2MASS (Skrutskie et al. 2006), UHS ${ }^{20}$ (Dye et al. 2018), and VHS (McMahon et al. 2013) J-band photometry. We also took into consideration photometric follow-up that our team has obtained for a relatively small subset of the full Backyard Worlds discovery list. Finally, when J-band imaging is available from UHS or VHS but no $\mathrm{J}$-band counterpart is present, we compute $5 \sigma$ depth limits at the relevant sky location according to the procedure described in Section 4.1 of Schneider et al. (2020). This allows us to identify esdT candidates on the basis of large $\mathrm{J}-\mathrm{W} 2$ color lower limits, even when a $\mathbf{J}$ detection is not available.

In our fiducial esdT candidate color-color selection box defined by $\mathrm{J}-\mathrm{W} 2>3 \mathrm{mag}$ and $1.1 \mathrm{mag}<\mathrm{W} 1-\mathrm{W} 2<1.75$ mag (see Figure 1), we found three new esdT candidates that remained viable after visual inspection to weed out problematic photometry (e.g., from WISE blending). We also required a W1 SNR of at least 10 in order to ensure that the measured W1 -W2 color is reliable. In Section 4, we discuss each of our three photometrically selected $\mathrm{T}$ subdwarf candidates in detail.

\footnotetext{
${ }^{20}$ The UKIDSS project is defined in Lawrence et al. (2007). UKIDSS uses the UKIRT Wide Field Camera (WFCAM; Casali et al. 2007) and a photometric system described in Hewett et al. (2006). The pipeline processing and science archive are described in Irwin (2008) and Hambly et al. (2008). We have used data from UHS DR1, which is described in detail in Dye et al. (2018).
} 


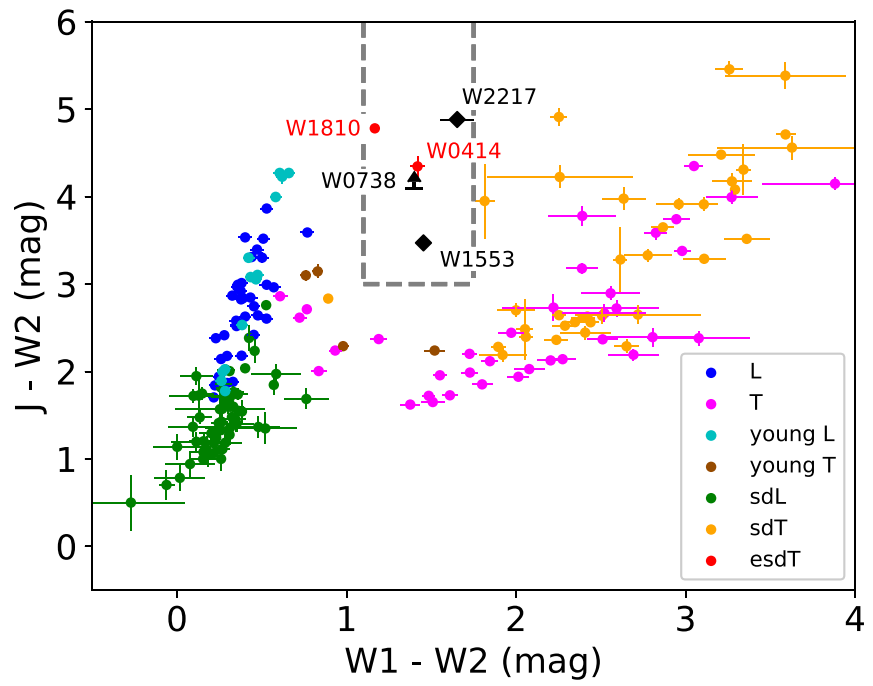

Figure 1. J-W2 vs. W1-W2 color-color plot showing the populations of L dwarfs (blue), T dwarfs (magenta), young L dwarfs within 20 pc (cyan; Kirkpatrick et al. 2021), young isolated T dwarfs (brown), L subdwarfs (green), $\mathrm{T}$ subdwarfs (orange), and extreme $\mathrm{T}$ subdwarfs (red). The two known esdT objects from Schneider et al. (2020) are individually labeled. WISEA 1553 +6933 and CWISE 2217-1454 are labeled with black diamonds. CWISE 0738-6643, a new esdT candidate first presented in this work, is labeled with an upward arrow to indicate that its nondetection in VHS provides a lower limit of $\mathrm{J}-\mathrm{W} 2=4.09 \mathrm{mag}$. WISEA $1553+6933$ and CWISE $2217-1454$ inhabit unusual regions of this color-color space, suggesting that they could be either sdT or esdT. CWISE 0738-6643 seems to most closely align with the esdTs Our adopted esdT candidate color-color selection box is shown as a gray dashed outline.

\section{T Subdwarf Candidates}

Figure 2 illustrates the large proper motions $(\mu \sim 0$ !" $9-$ 2 ". $2 \mathrm{yr}^{-1}$ ) of our three new subdwarf candidates as seen in WISE. The following subsections provide additional details about each subdwarf candidate's photometry and kinematics.

\subsection{CWISE J073844.52-664334.6}

CWISE 0738-6643 was discovered by Backyard Worlds participant Léopold Gramaize. CWISE 0738-6643 has W1 $-\mathrm{W} 2=1.40 \pm 0.05 \mathrm{mag}$ and is undetected in J-band imaging from VHS, yielding a J-W2 $>4.09$ mag color limit. In the Figure 1 color-color plot, its J-band lower limit places it at a location nearly identical to that of the esdT WISEA 0414 -5854 (Schneider et al. 2020). CWISE 0738-6643 is at least 2.15 magnitudes redder in $\mathrm{J}-\mathrm{W} 2$ than would be expected for a typical T dwarf of its same $\mathrm{W} 1-\mathrm{W} 2$ color. Table 1 provides photometric and astrometric information for CWISE 0738 -6643 . Using VHS imaging, we find a $5 \sigma$ limit of $K_{S}>18.22$ mag, but this limit is not deep enough to meaningfully constrain the nature of CWISE 0738-6643.

In addition to falling within our esdT candidate color-color selection box, CWISE 0738-6643 has a large total proper motion of $\mu=878 \pm 31$ mas $\mathrm{yr}^{-1}$ and a high W2 reduced proper motion $^{21}\left(H_{W 2}\right)$ similar to the largest $H_{W 2}$ values among the prior literature's population of spectroscopically confirmed T subdwarfs (see Figure 3). The compilations of literature sdT and sdL objects we use throughout this work were initially curated as part of the Schneider et al. (2020) analysis, and are

\footnotetext{
21 Defined as $H_{W 2}=m_{W 2}+5 \log _{10} \mu+5$, where $\mu$ is the total proper motion in arcseconds per year and $m_{W 2}$ is the W2 apparent magnitude (Luyten 1922).
}

largely based on lists from Zhang et al. (2019) and Zhang et al. (2018).

As none of our subdwarf candidates in this work have trigonometric parallax measurements available, reduced proper motion is a particularly valuable indicator pointing toward high kinematics and/or low luminosity. The reduced proper motion formula is a variant of the equation defining absolute magnitude, where the parallax has been replaced by total proper motion, in essence using large motion as a proxy for nearness. Because reduced proper motion grows with both apparent magnitude and total proper motion, objects with very low luminosity and/or high tangential velocity tend to have distinctively large reduced proper motions. Without trigonometric parallaxes for our subdwarf candidates, we cannot determine the extent to which their very high reduced proper motions are attributable to low luminosity versus high kinematics.

Using the Kirkpatrick et al. (2021) polynomial relations fit to the bulk T-dwarf population, we can derive WISE-based phototype and distance estimates for CWISE 0738-6643. From the W1-W2 color, we obtain a spectral type estimate of $\mathrm{T} 5 \pm 1.2$, and a photometric distance estimate of $43.6_{-8.4}^{+10.4} \mathrm{pc}$ (based on $M_{W 2}$ inferred from the WISE color). This photometric distance yields a $V_{\tan }$ estimate of $181_{-36}^{+44} \mathrm{~km} \mathrm{~s}^{-1}$. The large uncertainties on these values are primarily driven by the 0.46 mag scatter associated with the conversion from $\mathrm{W} 1-\mathrm{W} 2$ color to absolute magnitude (Kirkpatrick et al. 2021). If CWISE $0738-6643$ is subluminous at W2 relative to typical T dwarfs of the same W1-W2 color, its distance and tangential velocity will both be smaller than the estimates reported here. ${ }^{22}$

The CWISE 0738-6643 total proper motion value and its uncertainty from Table 1 both impact our $V_{\tan }$ estimate. Thanks to the fact that CatWISE 2020 spans a long $\sim 9 \mathrm{yr}$ time baseline and incorporates $6 \mathrm{yr}$ of WISE/NEOWISE imaging, it delivers a high $\mathrm{S} / \mathrm{N}$ proper motion measurement despite the faintness of CWISE 0738-6643 and the low WISE angular resolution $(\mathrm{FWHM} \approx 6$ ". 5). Marocco et al. (2021) compared the CatWISE 2020 proper motion measurements against those of Gaia DR2 (Gaia Collaboration et al. 2018), to assess the CatWISE 2020 proper motion accuracy and quoted uncertainties. CWISE 0738 -6643 is at very high absolute ecliptic latitude $\left(\beta=-80^{\circ} .3\right)$, meaning that Figure 23 of Marocco et al. (2021), which studies a region surrounding the south ecliptic pole, is the most applicable Gaia-CatWISE comparison. Figure 23 (bottom right panel) of Marocco et al. (2021) indicates that, near the south ecliptic pole, the median $\chi^{2}$ of the CatWISE 2020 versus Gaia DR2 proper motion comparison is $\sim 2$ for both $\mu_{\alpha}$ and $\mu_{\delta}$ at $\mathrm{W} 1=17.2 \mathrm{mag}$ (the CWISE 0738-6643 W1 magnitude). The median $\chi^{2}$ expected in this case (one degree of freedom) if the CatWISE motion uncertainties were correctly estimated would be 0.45 , so the measured median $\chi^{2}$ of $\sim 2$ indicates that the CatWISE motion uncertainties near the south ecliptic pole at $\mathrm{W} 1=17.2 \mathrm{mag}$ are typically underestimated by a factor of $\sqrt{2 / 0.45}=2.1$.

According to the quoted CatWISE 2020 motion uncertainties, the CWISE 0738-6643 total proper motion is measured with a $1 \sigma$ fractional error of $3.5 \%$. Given that the CWISE 0738 -6643 photometric distance uncertainty is at least $\sim 20 \%$, the proper motion contribution to the total $V_{\tan }$ uncertainty is very

\footnotetext{
22 The luminosity sequence of $\mathrm{T}$ subdwarfs is presently not well-mapped. L subdwarfs do exhibit different luminosity versus type trends than field L dwarfs in certain photometric bands (e.g., Gonzales et al. 2018).
} 

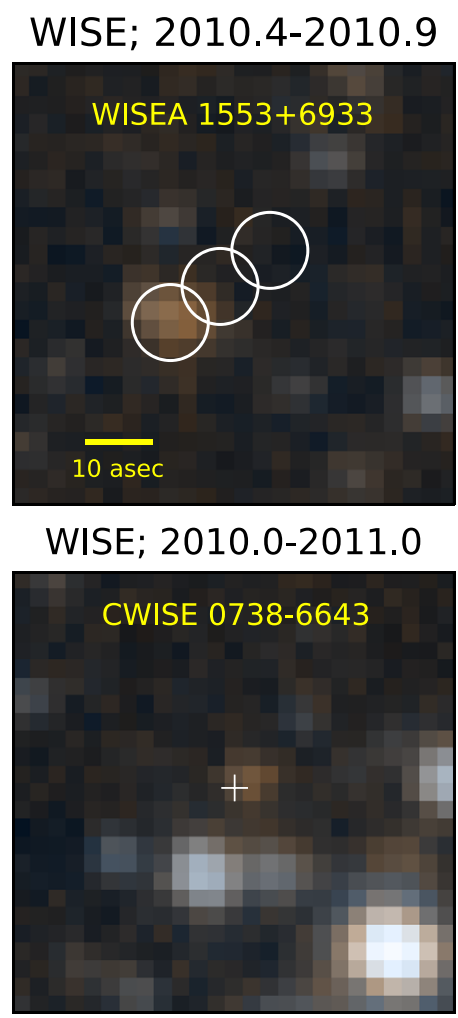

WISE; 2010.4-2010.9

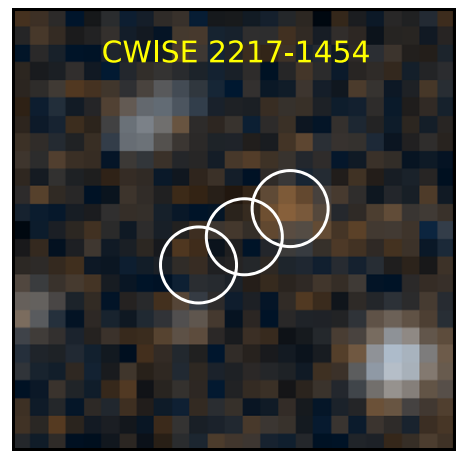

WISE; 2014.4-2014.9

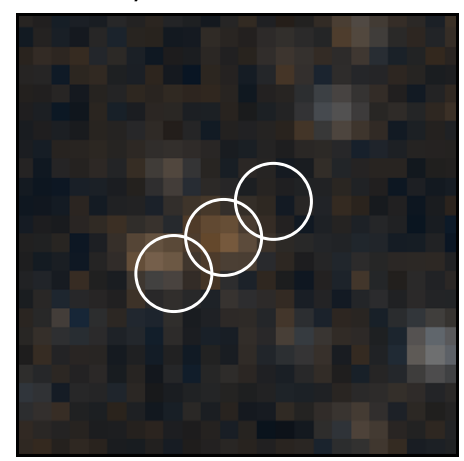

WISE; 2014.0-2014.5

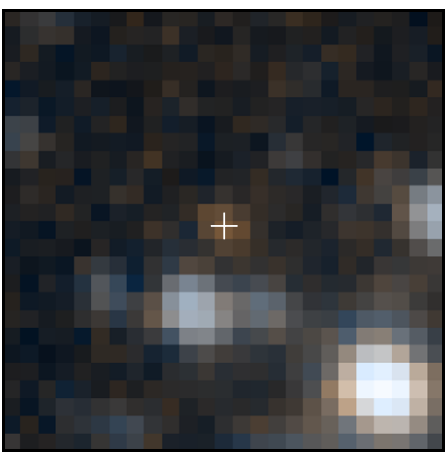

WISE; 2015.4-2015.9

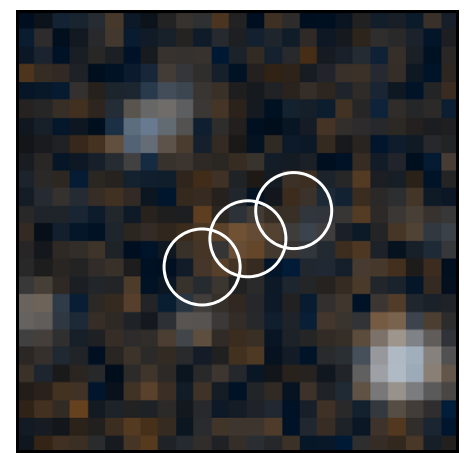

Spitzer; 2018.7

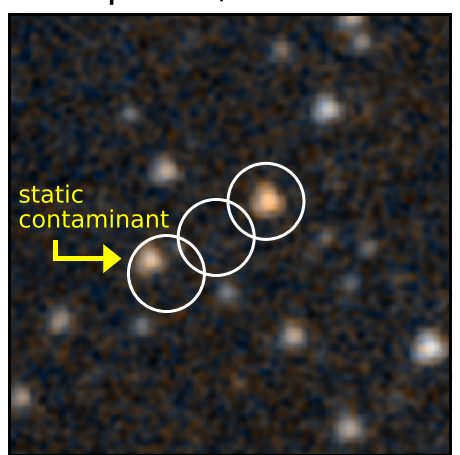

WISE; 2019.0-2019.5

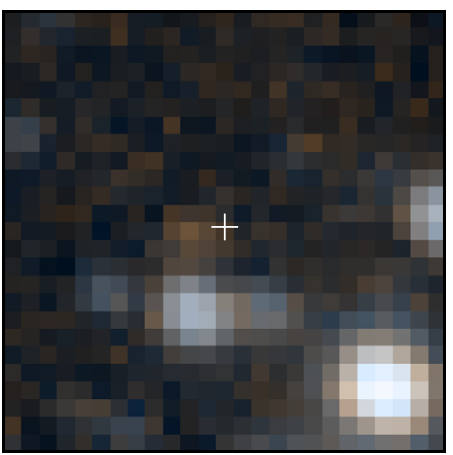

WISE; 2019.4-2019.8

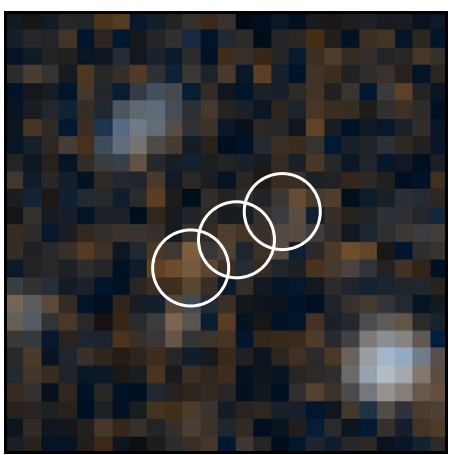

Figure 2. Top row: Time series of WISE and Spitzer images illustrating the motion of WISEA 1553+6933. Each panel is a two-band color composite rendering. In each case, W1 (ch1) is represented by the blue color channel and W2 (ch2) is represented by the red color channel. WISE images are one-year coadds spanning calendar 2010 (left) and the first year of NEOWISE-Reactivation (center). The Spitzer color composite at right is built from PID14076 IRAC imaging (PI: Faherty). East is left and north is up. The white circles track the northwesterly motion of WISEA 1553+6933 over the $~ 2010-2019$ time period. As seen in the left panel, WISEA $1553+6933$ was severely blended with a static background object during 2010. Middle row: WISE coadds illustrating the motion of CWISE 0738-6643. The white plus mark remains fixed to highlight the subdwarf candidate's southeasterly motion. Bottom row: WISE coadds illustrating the motion of CWISE $2217-1454$. All postage stamps are $1 ! 1 \times 1 ! 1$ in angular extent.

Table 1

Photometric Subdwarf Candidate Discoveries

\begin{tabular}{|c|c|c|c|c|c|c|c|}
\hline CWISE Name & W1 & W2 & $\overline{J_{\mathrm{MKO}}}$ & $\overline{K_{S}}$ & $\mu_{\alpha}\left(\right.$ mas yr $\left.^{-1}\right)$ & $\mu_{\delta}\left(\right.$ mas yr $\left.^{-1}\right)$ & $\mu\left({\left.\text { mas } \mathrm{yr}^{-1}\right)}^{\prime}\right.$ \\
\hline J073844.52-664334.6 & $17.221 \pm 0.038$ & $15.821 \pm 0.036$ & $>19.92$ & $>18.22$ & $765 \pm 32$ & $-432 \pm 30$ & $878 \pm 31$ \\
\hline J221706.28-145437.6 & $17.428 \pm 0.078$ & $15.775 \pm 0.055$ & $20.66 \pm 0.02$ & $>18.20$ & $1637 \pm 65$ & $-919 \pm 63$ & $1878 \pm 65$ \\
\hline
\end{tabular}

Note. New discoveries identified as extreme T subdwarf candidates based on their photometry and large motions. WISE magnitudes (uncertainties) come from the w1mpro_pm, w2mpro_pm (w1sigmpro_pm, w2sigmpro_pm) columns of the CatWISE 2020 catalog (Marocco et al. 2021). Proper motions are from CatWISE 2020. Quoted $\mu_{\alpha}$ values incorporate the $\cos (\delta)$ factor. All magnitude limits are $5 \sigma . K_{S}$ limits are based on archival VHS imaging.

subdominant, meaning that the total $V_{\tan }$ uncertainty is relatively insensitive to the exact $\mu$ uncertainty adopted (for instance, inflating the Table $1 \mu$ uncertainty for CWISE 0738 -6643 by a factor of 2.1 would only increase the total $V_{\tan }$ uncertainty by $5 \%$ ).

\subsection{WISEA J155349.96+693355.2}

WISEA 1553+6933 (Meisner et al. 2020) was first discovered by Backyard Worlds participant Nikolaj Stevnbak Andersen on 2017 December 9 using the WiseView image 

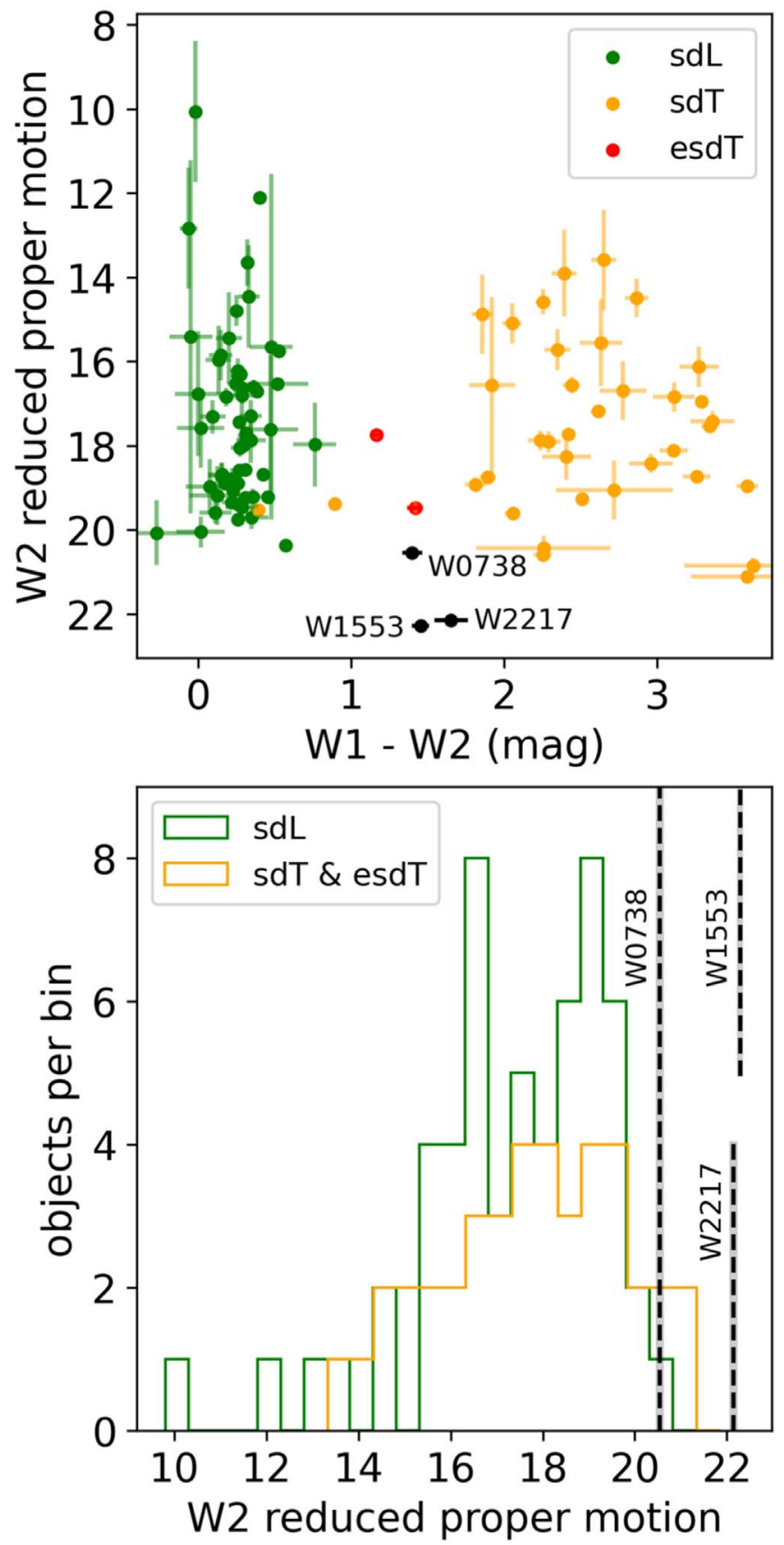

Figure 3. W2 reduced proper motions $\left(H_{W 2}\right)$ for this work's sample in comparison to the population of spectroscopically confirmed sdL, sdT, and esdT objects drawn from the prior literature. Top: $H_{W 2}$ as a function of W1-W2 color. Bottom: Histograms of sdL and sdT $H_{W 2}$ values with vertical dashed lines indicating the $H_{W 2}$ values of WISEA $1553+6933$, CWISE 2217-1454, and CWISE 0738 -6643 . WISEA $1553+6933$ has the largest W2 reduced proper motion among all spectroscopically confirmed $\mathrm{L}$ and $\mathrm{T}$ subdwarfs.

blinking tool. ${ }^{23}$ This object was later independently rediscovered by Backyard Worlds citizen scientist David W. Martin. Despite its large proper motion $\left(\mu=2157 \pm 55\right.$ mas $\left.\mathrm{yr}^{-1}\right)$, WISEA $1553+6933$ was missed by prior WISE-based brown dwarf searches, likely due to its faintness (W1 $=17.069 \pm 0.034$ mag, $\mathrm{W} 2=15.615 \pm 0.027 \mathrm{mag}$ ) and severe blending with a static background contaminant during the pre-hibernation WISE mission phase in 2010 (see Figure 2, top row).

${ }^{23}$ http://byw.tools/wiseview-v2
Table 2

WISEA J155349.96+693355.2

\begin{tabular}{lcc}
\hline \hline Parameter & Value & Ref. \\
\hline Observed Properties & & \\
$\mu_{\alpha}\left(\mathrm{mas} \mathrm{yr}^{-1}\right)$ & $-1684 \pm 56$ & 1 \\
$\mu_{\delta}\left(\mathrm{mas} \mathrm{yr}^{-1}\right)$ & $1348 \pm 53$ & 1 \\
$z_{A B}(\mathrm{mag})$ & $22.17 \pm 0.21$ & 3 \\
$\mathrm{~J}_{\mathrm{MKO}}(\mathrm{mag})$ & $19.086 \pm 0.065$ & 2 \\
W1 $(\mathrm{mag})$ & $17.069 \pm 0.034$ & 2 \\
W2 $(\mathrm{mag})$ & $15.615 \pm 0.027$ & 2 \\
ch1 $(\mathrm{mag})$ & $16.324 \pm 0.019$ & 1 \\
ch2 $(\mathrm{mag})$ & $15.458 \pm 0.018$ & 1 \\
Inferred Properties & & 2 \\
$T_{\text {eff }}(\mathrm{K})$ & $1200 \pm 100$ & 2 \\
$\log (g)$ & $\approx 5.0-5.5$ & 2 \\
{$[\mathrm{Fe} / \mathrm{H}](\mathrm{dex})$} & $\approx-0.5$ & \\
\hline
\end{tabular}

References. (1) Meisner et al. 2020; (2) This work; (3) Nidever et al. 2018.

Table 2 lists the available photometric and astrometric properties for WISEA $1553+6933$. Because WISEA 1553 +6933 was blended during 2010, we opted to perform custom W1 and W2 photometry rather than use AllWISE (Cutri et al. 2013) or CatWISE (Eisenhardt et al. 2020; Marocco et al. 2021) photometry. Our custom photometry was obtained by running the crowdsource pipeline (Schlafly et al. 2018, 2019) on a set of coadded unWISE images (Meisner et al. 2018), each of which stacks together a single WISE sky pass worth of exposures. There are 12 such coadded epochs available per band spanning 2014 to 2019 , and the results of averaging the per sky pass W1 and W2 crowdsource photometry are provided in Table 2 . The Spitzer ch1 $(3.6 \mu \mathrm{m})$ and ch2 $(4.5 \mu \mathrm{m})$ photometry in Table 2 is from PID14076 (PI: Faherty; Meisner et al. 2020). WISEA 1553+6933 was also detected at low significance by the Mayall $z$-band Legacy Survey (MzLS; Dey et al. 2019) with $z_{\mathrm{AB}}=22.17 \pm 0.21$ mag (Nidever et al. 2018), though no brown dwarf absolute magnitude or color-type relations incorporating Mayall/Mosaic3 (Dey et al. 2016) $z$-band currently exist, limiting the utility of this $z$-band data point.

WISEA $1553+6933$ is undetected by 2 MASS and too far north for UKIRT imaging. Meisner et al. (2020) obtained a $5 \sigma$ MKO J-band limit of J > 17.34 mag using the CPAPIR imager (Artigau et al. 2004) at the Mont Megantic Observatory (Racine 1978). The corresponding $\mathrm{J}-\mathrm{W} 2>1.73 \mathrm{mag}$ color limit is consistent with either a T-dwarf or T-subdwarf scenario. We therefore sought deeper J-band imaging to better constrain the nature of WISEA $1553+6933$ and assess the prospects for near-infrared spectroscopy. On 2018 March 29, we obtained $25 \times 60$ s MKO J-band exposures (PI: Wisniewski) of WISEA 1553+6933 with the NIRI instrument (Hodapp et al. 2003) on the 8.1 meter Gemini North telescope. The J-band delivered image quality was $0 . \prime 6$ and conditions were photometric. We reduced the Gemini J-band images with $\mathrm{IRAF}^{24}$ and calibrated the photometric zero point to 2MASS after converting 2MASS calibrator $\mathrm{J}$ magnitudes to the MKO system. The uncertainty on our Gemini J-band magnitude in Table 2 is dominated by the tie-down to 2MASS, as a limited number of nearby 2MASS calibrators were available and these have only modest $\mathrm{S} / \mathrm{N}$ in 2MASS. WISEA $1553+6933$ is 1.54 (1.75) magnitudes redder in $\mathrm{J}-\mathrm{W} 2(\mathrm{~J}-\mathrm{ch} 2)$

\footnotetext{
$\overline{24}$ http://ast.noao.edu/sites/default/files/A_Guide_to_Reducing_Near-IR_ Images_1.pdf
} 
color than would be expected for a typical T dwarf of its same $\mathrm{W} 1-\mathrm{W} 2$ (ch1-ch2) color.

The Spitzer-based photometric distance estimate of $38.6_{-4.2}^{+4.6} \mathrm{pc}$ for WISEA $1553+6933$ from Meisner et al. (2020) implies an extremely high tangential velocity of $V_{\tan }=395_{-44}^{+48} \mathrm{~km} \mathrm{~s}^{-1}$. The WISEA $1553+6933$ phototype based on Spitzer ch1-ch2 color is $\mathrm{T} 5 \pm 1$ (Meisner et al. 2020) and the polynomial relations from Table 13 of Kirkpatrick et al. (2021) yield a Spitzer-based temperature estimate of $1076 \pm 89 \mathrm{~K}$. Note that the Spitzer-based photometric distance and temperature relations are fit to "normal" T dwarfs. If WISEA $1553+6933$ is subluminous at ch2 relative to typical $\mathrm{T}$ dwarfs of the same ch1-ch2 color, its distance and tangential velocity will both be lower than the estimates reported here. It would be surprising if the WISEA $1553+6933 V_{\tan }$ were truly so high as $\sim 400 \mathrm{~km} \mathrm{~s}^{-1}$, although it is the case that our motion selection methodology biases us toward finding objects with preferentially high $V_{\text {tan }}$. For example, the parent Spitzer sample from which WISEA $1553+6933$ is drawn (Meisner et al. 2020) has a median estimated $V_{\tan }$ of $60 \mathrm{~km} \mathrm{~s}^{-1}$, which is $\sim 2 \times$ higher than the median $V_{\tan }$ of late $\mathrm{T}$ and $\mathrm{Y}$ dwarfs within 20 pc from Kirkpatrick et al. (2019b). WISEA $1553+6933$ has $H_{W 2}=22.28 \pm 0.06 \mathrm{mag}$, a larger $\mathrm{W} 2$ reduced proper motion than that of any spectroscopically confirmed $\mathrm{L}$ or $\mathrm{T}$ subdwarf from the prior literature, ${ }^{25}$ by a margin of $\sim 1.2$ magnitudes (see Figure 3).

\subsection{CWISE J221706.28-145437.6}

CWISE 2217-1454 was initially discovered by Dan Caselden using supervised machine-learning methods to identify fast-moving, red objects based on proper motions and W1-W2 colors drawn from the CatWISE 2020 catalog (see Section 3 of Marocco et al. 2019 for details of this selection methodology). CWISE 2217-1454 was also independently discovered by Backyard Worlds citizen scientists Léopold Gramaize, Sam Goodman, and Arttu Sainio.

CWISE 2217-1454 has $\mathrm{W} 1-\mathrm{W} 2=1.65 \pm 0.10 \mathrm{mag}$ and is undetected in J-band imaging from VHS. To probe deeper in the near-infrared, we obtained follow-up Keck/MOSFIRE (McLean et al. 2012) J-band imaging on the night of 2020 September 3 (PI: Marocco). Observing conditions were excellent, with clear sky and $\sim 0$." 5 seeing. We acquired $18 \times 100 \mathrm{~s}$ frames which were subsequently coadded and photometrically calibrated to 2MASS after converting the 2MASS calibrator J magnitudes to the MKO system. We find a high-significance detection of CWISE 2217 $-1454: \mathrm{J}=20.66 \pm 0.02 \mathrm{mag}$. With $\mathrm{J}-\mathrm{W} 2=4.88 \pm 0.06 \mathrm{mag}$, CWISE 2217-1454 is thus 2.93 magnitudes redder in $\mathrm{J}-\mathrm{W} 2$ than would be expected for a typical T dwarf of its same W1-W2 color (Kirkpatrick et al. 2021). Using VHS imaging, we find a $5 \sigma$ limit of $K_{S}>18.20 \mathrm{mag}$, but this limit is not deep enough to materially constrain the nature of CWISE 2217-1454.

Photometric and astrometric properties of CWISE 2217 -1454 are listed in Table 1 . In addition to its anomalously red $\mathrm{J}$ -W2 color, CWISE $2217-1454$ has a very large total proper motion of $\mu=1878 \pm 65$ mas $\mathrm{yr}^{-1}$. Its W2 reduced proper motion of $H_{W 2}=22.14 \pm 0.09 \mathrm{mag}$ is larger than that of any spectroscopically confirmed L or $\mathrm{T}$ subdwarf from the prior literature, and only $\sim 0.15 \mathrm{mag}$ lower than that of WISEA 1553 +6933 (see Figure 3).

\footnotetext{
25 Note, however, that WISEA $1553+6933$ does not have the largest W2 reduced proper motion among all known brown dwarfs. For instance, the Y dwarf WISE $0855-0714$ (Luhman 2014) has $H_{W 2} \approx 23.6$ mag.
}

Using the Kirkpatrick et al. (2021) polynomial relations fit to the bulk $\mathrm{T}$ dwarf population, we can derive WISE-based phototype and distance estimates for CWISE 2217-1454. From the W1-W2 color, we obtain a spectral type estimate of $\mathrm{T} 5.5 \pm 1.2$ and a photometric distance estimate of $39.4_{-7.6}^{+9.5} \mathrm{pc}$ (based on $M_{W 2}$ inferred from the WISE color). This photometric distance estimate yields a corresponding $V_{\tan }$ estimate of $351_{-69}^{+85} \mathrm{~km} \mathrm{~s}^{-1}$. As for CWISE 0738-6643, if CWISE 2217 -1454 is subluminous at WISE wavelengths relative to typical $\mathrm{T}$ dwarfs of the same W1-W2 color, its distance and tangential velocity will both be smaller than the present estimates. The CWISE 2217-1454 proper motion uncertainties quoted in Table 1 may be underestimated by $\sim 7 \%$ based on Figure 13 of Marocco et al. (2021), which provides the most applicable assessment of the quoted CatWISE 2020 motion uncertainties for CWISE 2217-1454, given that CWISE 2217-1454 is near the ecliptic plane rather than the south ecliptic pole. Inflating the CWISE 2217-1454 proper motion uncertainties by $\sim 7 \%$ would negligibly increase our quoted total $V_{\tan }$ uncertainty, as the latter is strongly dominated by the large $\sim 20 \%$ uncertainty on our photometric distance estimate.

\section{Spectroscopy}

We used the Near-Infrared Echellette Spectrometer (NIRES; Wilson et al. 2004) on the Keck II telescope on 2020 July 7 (UT) to obtain $0.94-2.45 \mu \mathrm{m}$ near-infrared spectra of WISEA $1553+6933$. Conditions were clear with 0.9 seeing. Keck/ NIRES has a fixed instrument configuration with a 0 ".55 slit producing resolution $\sim 2700$ data. The target was visible in the K-band slit-viewing camera and placed into the spectroscopic slit. We obtained a set of $12 \times 300$ second spectroscopic frames obtained in an ABBA nodding pattern along the slit over an airmass range of 2.0-2.4. The A0 V star TYC 4282-488-1 $(V=10.83 \mathrm{mag}$ ) was observed immediately afterward for flux calibration and telluric correction, and flat-field lamp exposures were obtained for pixel response calibration. Data were reduced using a modified version of Spextool (Cushing et al. 2004; see also Section 4.4 of Kirkpatrick et al. 2011), following the standard procedure, which includes: pixel calibration, wavelength calibration, and spatial and spectral rectification using flat-field and telluric line exposures; optimal extraction of individual spectra from A-B pairwise subtracted frames; combination of these spectra with outlier masking; and telluric correction using the A0 V spectrum following Vacca et al. (2003). The reduced, smoothed Keck/NIRES spectrum is shown in Figures 4-6 in comparison to various standards, literature subdwarfs, and atmospheric models.

The left column of Figure 4 compares the WISEA 1553 +6933 spectrum against T3 (2MASS J12095613-1004008), T4 (2MASSI J2254188+312349), and T5 (2MASS J15031961 +2525196) standards from the SpeX Prism Library (Burgasser et al. 2006; Burgasser 2014). All spectra in Figure 4 are normalized to unity between $1.27 \mu \mathrm{m}$ and $1.29 \mu \mathrm{m}$. The T4 standard provides the best visual match: the WISEA 1553 +6933 spectrum at wavelengths of $1-1.3 \mu \mathrm{m}$ is replicated reasonably well by the $\mathrm{T} 4$ standard. In the $\mathrm{H}$ band, WISEA $1553+6933$ appears broader than the T4 standard, with the observed H-band peak extending bluer than that of the T4 standard. Relative to the T4 standard, neither the T3 nor T5 standard better matches WISEA $1553+6933$ at $\mathrm{H}$ band; the T3 standard is too elevated and broad in $\mathrm{H}$ band, while the T5 standard has an insufficiently strong, overly narrow H-band 

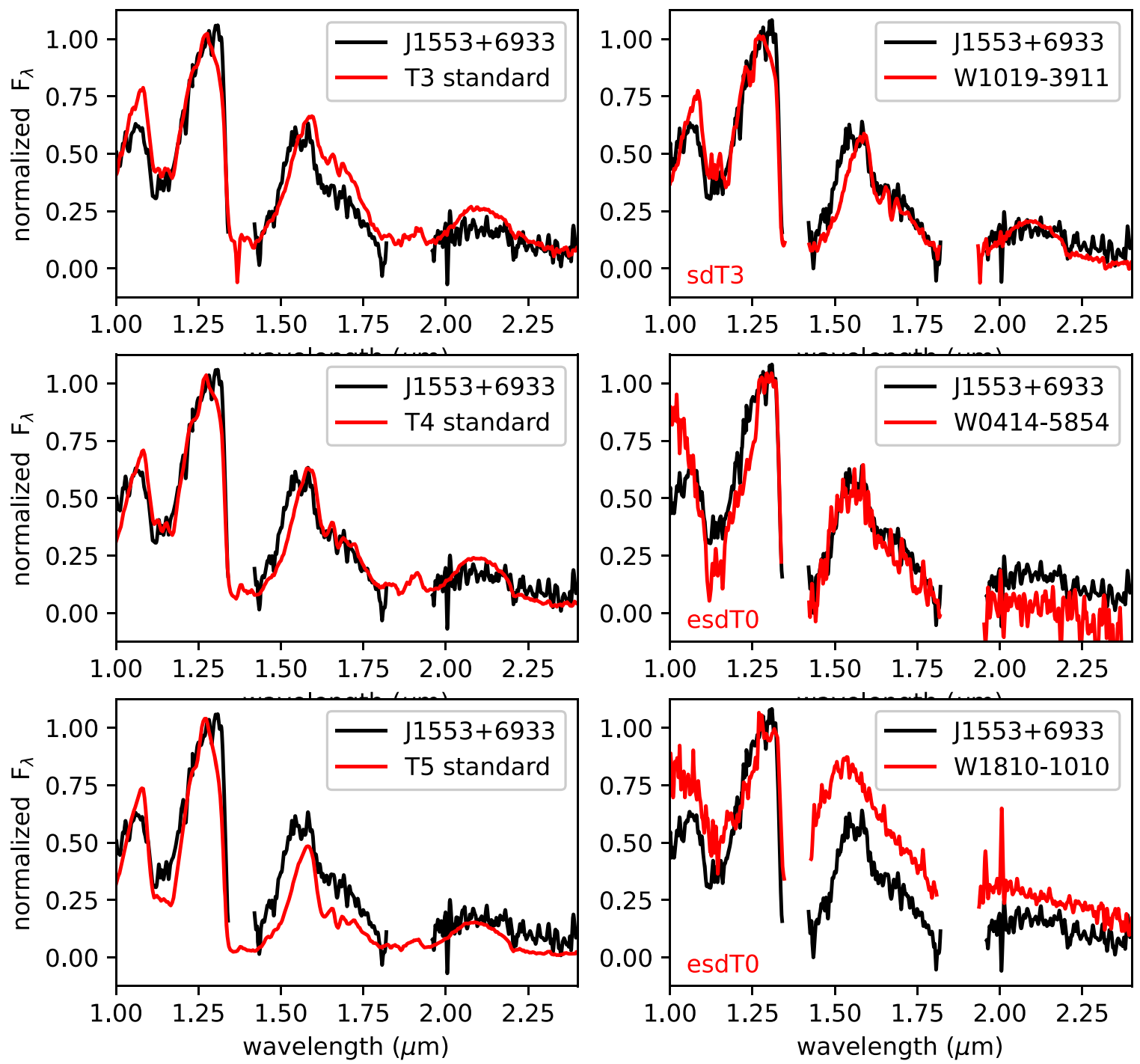

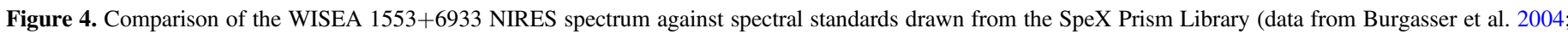

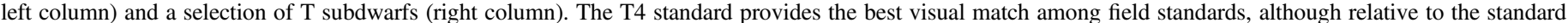

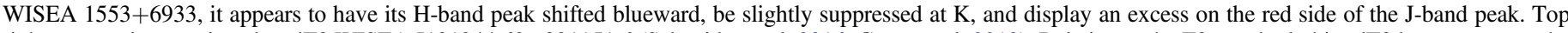

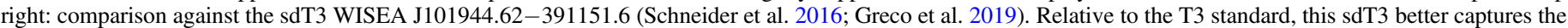

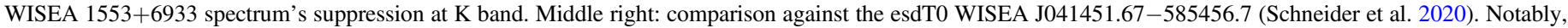

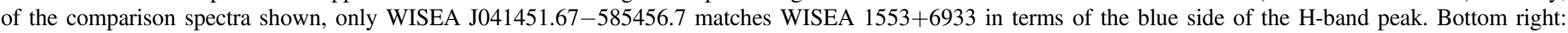
comparison against the esdT0 WISEA J181006.18-101000.5 (Schneider et al. 2020). All spectra are normalized to unity between $1.27 \mu$ m and $1.29 \mu$ m.

peak. At $\mathrm{K}$ band, the T4 again provides the best match among the standards, although the WISEA $1553+6933$ spectrum appears "flatter" than all three T standards in the $2-2.4 \mu \mathrm{m}$ wavelength range. Such K-band suppression is expected for T subdwarfs due to collision-induced $\mathrm{H}_{2}$ absorption, which becomes enhanced when metallicity is low and surface gravity is high (e.g., Saumon et al. 2012). We also note that, relative to the T standards, WISEA J1553+6933 displays a flux excess on the red side of its J-band peak, a hallmark feature in the spectra of T subdwarfs (Mace et al. 2013b). This subdwarf signature in the WISEA $1553+6933$ spectrum is not well-replicated by any of our best-fitting models (Figures 5 and 6). Our finding that, among the standards, T4 best matches WISEA $1553+6933$ is consistent with its Spitzer-based phototype of $\mathrm{T} 5 \pm 1$ from Meisner et al. (2020).

Figure 4 also provides a comparison against the sdT3 WISEA J101944.62-391151.6 (Schneider et al. 2016; Greco et al. 2019, hereafter WISEA 1019-3911). At K band, the
WISEA 1553+6933 spectrum's amplitude and shape are slightly better matched by WISEA 1019-3911 than by any of the T-dwarf standards. Like the T standards, WISEA 1019 -3911 also fails to match the H-band peak shape observed for WISEA 1553+6933. Interestingly, the esdT WISEA 0414 -5854 (Schneider et al. 2020) matches WISEA $1553+6933$ better at $\mathrm{H}$ band than either the sdT WISEA 1019-3911 or the $\mathrm{T}$ standards. The other esdT plotted (WISEA 1810-1010; Schneider et al. 2020) is dramatically enhanced at $\mathrm{H}$ band in a manner that does not match WISEA $1553+6933$. The esdT spectra also exhibit major differences relative to WISEA 1553 +6933 blueward of $1.3 \mu \mathrm{m}$. In particular, the esdTs show a strong flux enhancement at $\sim 1-1.1 \mu \mathrm{m}$ not present in the WISEA $1553+6933$ spectrum. The esdT K-band spectra are poor matches to WISEA $1553+6933$ : WISEA $0414-5854$ is much suppressed whereas WISEA $1810-1010$ is significantly enhanced. 
PHOENIX models
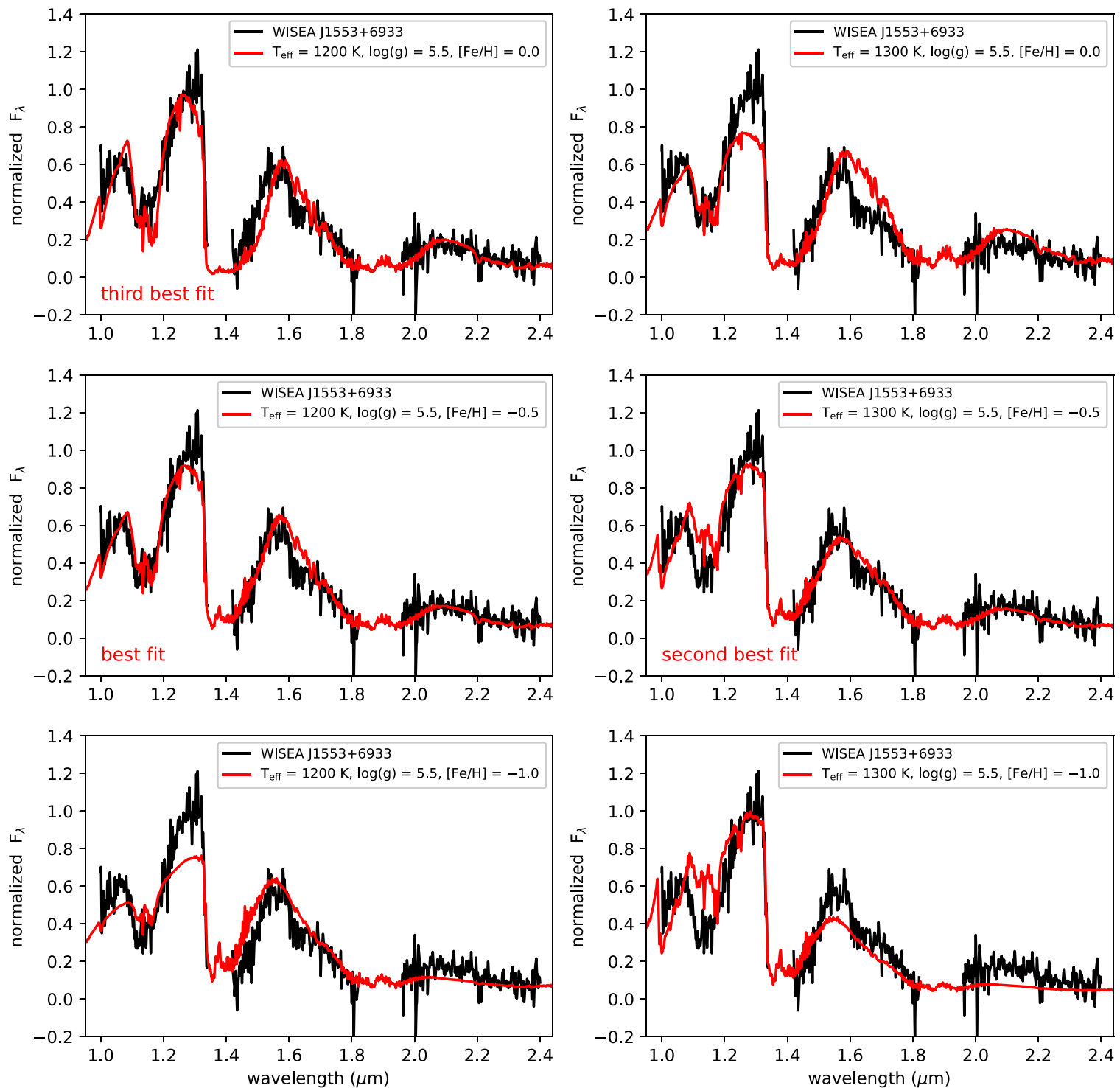

Figure 5. Comparison of the WISEA $1553+6933$ NIRES spectrum against various models from a PHOENIX model atmosphere grid extending to low metallicity (Gerasimov et al. 2020). The top row's models have solar metallicity, the middle row's models have $[\mathrm{Fe} / \mathrm{H}]=-0.5$, and the bottom row's models have $[\mathrm{Fe} / \mathrm{H}]=-1.0$. The left column's models have $T_{\text {eff }}=1200 \mathrm{~K}$, while the right column's models have $T_{\text {eff }}=1300 \mathrm{~K}$. The best-fit model has $[\mathrm{Fe} / \mathrm{H}]=-0.5$ and $T_{\text {eff }}=1200 \mathrm{~K}$. The second (third) best-fit model has $[\mathrm{Fe} / \mathrm{H}]=-0.5([\mathrm{Fe} / \mathrm{H}]=0)$ and $T_{\text {eff }}=1300 \mathrm{~K}\left(T_{\text {eff }}=1200 \mathrm{~K}\right)$.

In Sections 5.1 and 5.2, we fit the WISEA $1553+6933$ NIRES spectrum with two grids of low-metallicity model atmosphere spectra in order to quantify the metallicity and effective temperature of WISEA $1553+6933$.

\subsection{PHOENIX Models}

We compared the WISEA $1553+6933$ spectrum against a set of PHOENIX atmospheric models (Hauschildt \& Baron 1999; Allard et al. 2013) extending to low metallicity ${ }^{26}$ (Gerasimov et al. 2020). In total, this PHOENIX model grid contains 44 spectra ranging in metallicity from $[\mathrm{Fe} / \mathrm{H}]=0$ to $[\mathrm{Fe} / \mathrm{H}]=$ -3 dex and from 900 to $1500 \mathrm{~K}$ in effective temperature, with two $\log (g)$ values available (5.0 and $5.5 ; g$ in cgs units). Each model spectrum covers the $0.4-2.6 \mu \mathrm{m}$ wavelength range. The

${ }^{26} \mathrm{http}: / /$ atmos.ucsd.edu/?p=atlas $\log (g)=5.0$ PHOENIX models all provide very poor fits to the WISEA $1553+6933$ spectrum. This PHOENIX grid is rather sparse; for instance, the grid's native metallicity spacing is 1 dex. Overlaying the $\log (g)=5.5$ models at $T_{\text {eff }}=1200-1300 \mathrm{~K}$ as in Figure 5, it immediately became clear that this would be the best-fit temperature range for WISEA $1553+6933$ and that the optimal metallicity would lie somewhere between $[\mathrm{Fe} / \mathrm{H}]=-1$ and $[\mathrm{Fe} / \mathrm{H}]=0$. We therefore interpolated the PHOENIX grid in metallicity to provide model templates at half-dex intervals. This interpolation is one-dimensional in the sense that we only interpolate between a pair of models separated by 1 dex in $[\mathrm{Fe} / \mathrm{H}]$ when those two models are identical in all other parameters, including $T_{\text {eff }}$ and $\log (g)$. This interpolation procedure results in 25 additional models with half-integer metallicity values of $[\mathrm{Fe} / \mathrm{H}]=-0.5,-1.5$, and -2.5 dex. As an example, in the left column of Figure 5, the middle panel's model $\left([\mathrm{Fe} / \mathrm{H}]=-0.5, T_{\text {eff }}=1200 \mathrm{~K}, \log \right.$ 


\section{LOWZ models, $\mathrm{T}_{\text {eff }}=1100 \mathrm{~K}$}
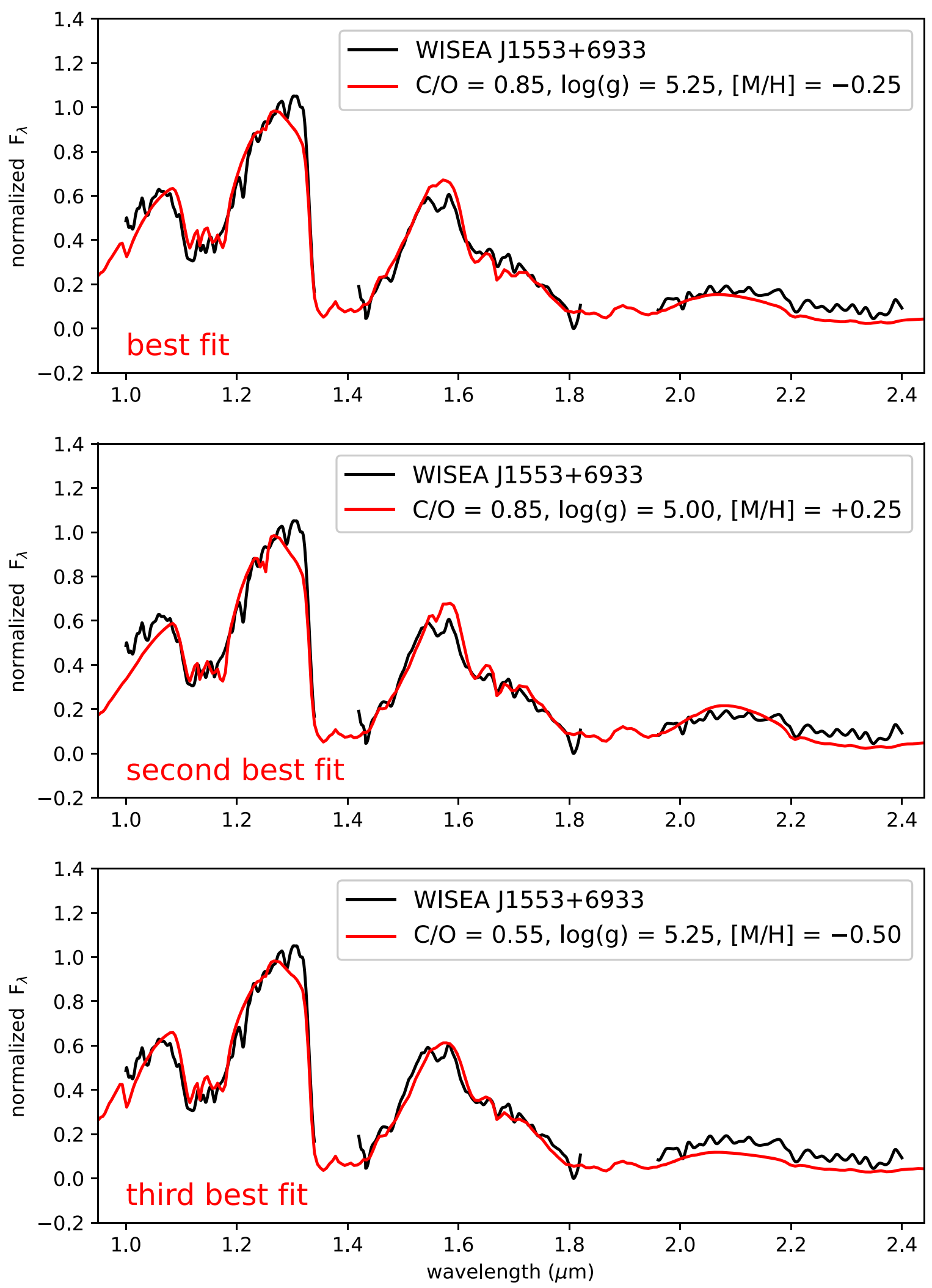

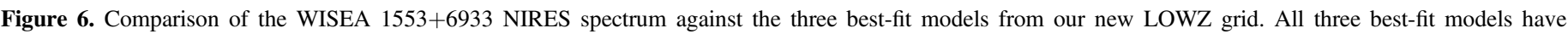

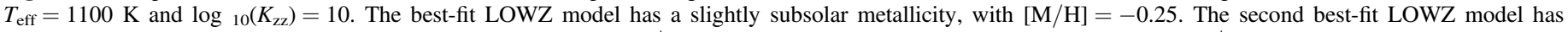
relatively $\operatorname{low} \log (g)=5.0$ and a slightly supersolar metallicity, $[\mathrm{M} / \mathrm{H}]=+0.25$ dex. The third best-fit LOWZ model has $[\mathrm{M} / \mathrm{H}]=-0.5$. 
$(g)=5.5)$ is an interpolation in metallicity between the model shown in the top panel of that column $\left([\mathrm{Fe} / \mathrm{H}]=0, T_{\text {eff }}=\right.$ $1200 \mathrm{~K}, \log (g)=5.5)$ and the model shown in the bottom panel of that column $\left([\mathrm{Fe} / \mathrm{H}]=-1, T_{\text {eff }}=1200 \mathrm{~K}, \log (g)=5.5\right)$. The same applies to the right column of Figure 5, except for the case of $T_{\text {eff }}=1300 \mathrm{~K}$.

We fit all 69 models in our augmented PHOENIX grid to the observed WISEA $1553+6933$ spectrum, using the $\chi^{2}$ goodnessof-fit metric. When fitting, the observed spectrum is normalized to unity at the J-band peak, and the overall normalization of each model (across the full 1-2.4 $\mu \mathrm{m}$ wavelength range) is treated as a single free parameter. Figure 5 shows a comparison of the WISEA $1553+6933$ spectrum against six PHOENIX models spanning the reasonably well-fitting range of temperature and metallicity: $1200 \mathrm{~K} \leqslant T_{\text {eff }} \leqslant 1300 \mathrm{~K},-1 \leqslant[\mathrm{Fe} / \mathrm{H}] \leqslant 0$ and $\log (g)=5.5$. The three best-fitting models are labeled in Figure 5. The best-fit model has $T_{\text {eff }}=1200 \mathrm{~K},[\mathrm{Fe} / \mathrm{H}]=-0.5 \mathrm{dex}$ and $\log (g)=5.5$ (left column, middle row of Figure 5). Among the set of three bestfitting models, two have $[\mathrm{Fe} / \mathrm{H}]=-0.5$, one has $[\mathrm{Fe} / \mathrm{H}]=0$, and all have $T_{\text {eff }}=1200 \mathrm{~K}$ or $1300 \mathrm{~K}$.

The $T_{\text {eff }}=1200 \mathrm{~K},[\mathrm{Fe} / \mathrm{H}]=0$ model mostly matches the WISEA 1553+6933 spectrum, but like the T4 standard (Section 5), this model fails to capture the observed H-band peak's blue extension. The $T_{\text {eff }}=1200 \mathrm{~K},[\mathrm{Fe} / \mathrm{H}]=-1$ model has a broadened H-band peak, but this model's H-band peak is much stronger relative to $\mathrm{J}$ and $\mathrm{K}$ than is observed for WISEA $1553+6933$. The best-fit model $\left(T_{\text {eff }}=1200 \mathrm{~K},[\mathrm{Fe} / \mathrm{H}]=-0.5\right)$ accurately matches the blue wing of the $\mathrm{H}$-band peak while also displaying the correct relative amplitudes for all three spectral segments between 1.0 and $2.4 \mu \mathrm{m}$. For $T_{\text {eff }}=1300 \mathrm{~K}$, the observed $\mathrm{H}$-band peak is again best reproduced with $[\mathrm{Fe} / \mathrm{H}]=-0.5$, but the agreement at $1-1.3 \mu \mathrm{m}$ is substantially worse throughout the $-1 \leqslant[\mathrm{Fe} / \mathrm{H}] \leqslant 0$ metallicity range.

\subsection{New LOWZ Models}

We have also generated an extensive new grid of lowtemperature, low-metallicity model atmosphere spectra and compared these against the near-infrared spectrum and broadband colors of WISEA $1553+6933$. Since we expect this new model grid, which we refer to as "LOWZ," to be generally useful for studies of brown dwarfs, we have made these models publicly available online in the Harvard Dataverse:https://doi. org/10.7910/DVN/SJRXUO.

We use the ScCHIMERA 1D-radiative-convective-thermochemical equilibrium tool (Arcangeli et al. 2018; Mansfield et al. 2018; Piskorz et al. 2018; Gharib-Nezhad \& Line 2019; Zalesky et al. 2019; Baxter et al. 2020; Beatty et al. 2020; Colón et al. 2020) to compute our cloud-free model grid. Briefly, the grid solves for the net radiative fluxes using the Toon et al. (1989) two-stream source function technique. The equilibrium temperature structure is achieved using a NewtonRaphson scheme (e.g., McKay et al. 1989). Chemical equilibrium is computed using the NASA Chemical Equilibrium with Applications ${ }^{27}$ routine assuming rainout chemistry and scaled Lodders \& Palme (2009) solar abundances. We include a suite of relevant opacities $\left(\mathrm{H}_{2}-\mathrm{H}_{2} / \mathrm{He}\right.$ collisioninduced absorption (Richard et al. 2012), $\mathrm{H}^{-}$bound-free/freefree (John 1988), $\mathrm{H}_{2} \mathrm{O}$ (Polyansky et al. 2018), CO (Rothman et al. 2010), $\mathrm{CH}_{4}$ (Yurchenko \& Tennyson 2014), $\mathrm{NH}_{3}$ (Yurchenko et al. 2011), $\mathrm{H}_{2} \mathrm{~S}$ (Tennyson \& Yurchenko 2012),

\footnotetext{
27 https://cearun.grc.nasa.gov/
}

$\mathrm{PH}_{3}$ (Sousa-Silva et al. 2015), $\mathrm{HCN}$ (Harris et al. 2008), $\mathrm{C}_{2} \mathrm{H}_{2}$ (Rothman et al. 2013), TiO (McKemmish et al. 2019), VO (McKemmish et al. 2016), $\mathrm{SiO}$ (Barton et al. 2013), $\mathrm{CaH}$ (Yadin et al. 2012; Alavi \& Shayesteh 2018), MgH (Yadin et al. 2012; GharibNezhad et al. 2013), CrH (Burrows 2005), AlH (Yurchenko et al. 2018), FeH (Wende et al. 2010), Na, and K (Allard et al. 2007, 2019)), as well as several other atomics sourced from Kurucz (1993) converted into $R=250$ correlated$\mathrm{K}$ coefficients (with 10 Gauss-quadrature points per bin, in double Gauss form) mixed on the fly using the Amundsen et al. (2017) resort-rebin procedure. Convection is treated as an additional flux via mixing length theory.

Each model in the LOWZ grid extends from $0.1-10 \mu \mathrm{m}$ in wavelength coverage. The wavelength grid spacing is $4.6 \mathrm{~nm}$ at $\lambda=1.15 \mu \mathrm{m}, 6.6 \mathrm{~nm}$ at $\lambda=1.65 \mu \mathrm{m}$, and $8.8 \mathrm{~nm}$ at $\lambda=2.2$ $\mu \mathrm{m}$. The LOWZ model metallicities range from $[\mathrm{M} / \mathrm{H}]=-2.5$ to +1.0 dex, sampling values of $[\mathrm{M} / \mathrm{H}]=-2.5,-2,-1.5,-1$, $-0.5,-0.25,0,+0.25,+0.5,+0.75$, and +1 dex. The LOWZ effective temperatures span from $T_{\text {eff }}=500 \mathrm{~K}$ to $T_{\text {eff }}=1600 \mathrm{~K}$, with values of $T_{\text {eff }}=500,550,600,650,700,750,800,850$, $900,950,1000,1100,1200,1300,1400,1500$, and $1600 \mathrm{~K}$. The grid contains three distinct carbon-to-oxygen $(\mathrm{C} / \mathrm{O})$ ratio values $(0.1,0.55,0.85)$, five $\log (g)$ values $(3.5,4,4.5,5,5.25)$, and three vertical eddy diffusion coefficient options, log ${ }_{10}\left(K_{\mathrm{zz}}\right)=(-1,2,10)$, with $K_{\mathrm{zz}}$ in units of $\mathrm{cm}^{2} \mathrm{~s}^{-1}$. In all, our LOWZ grid contains 8,402 model atmosphere spectra sampling in the five-dimensional space of $\left([\mathrm{M} / \mathrm{H}], T_{\mathrm{eff}}, \mathrm{C} / \mathrm{O}\right.$, $\left.\log (g), \log _{10}\left(K_{\mathrm{zz}}\right)\right)$.

We fit all 8402 LOWZ models to the observed WISEA 1553 +6933 spectrum, again using the $\chi^{2}$ goodness-of-fit metric. When fitting, the observed spectrum is normalized to unity at the J-band peak, and the overall normalization of each model (across the full $1-2.4 \mu \mathrm{m}$ wavelength range) is treated as a single free parameter. Figure 6 shows the WISEA 1553+6933 NIRES spectrum overlaid on the three best-fitting LOWZ models. All three best-fitting models have $T_{\text {eff }}=1100 \mathrm{~K}$ and $\log { }_{10}\left(K_{\mathrm{zz}}\right)=10$. The best-fit LOWZ model has a slightly subsolar metallicity, with $[\mathrm{M} / \mathrm{H}]=-0.25, \mathrm{C} / \mathrm{O}=0.85$, and $\log (g)=5.25$. The second best-fit LOWZ model is slightly metal-enhanced with $[\mathrm{M} / \mathrm{H}]=+0.25$ and $\operatorname{lower} \log (g)=5$ rather than 5.25. The third best-fit LOWZ model has $[\mathrm{M} / \mathrm{H}]=-0.5, \log (g)=5.25$ and a $\mathrm{C} / \mathrm{O}$ ratio of 0.55 .

One peculiarity of our best-fit LOWZ model's parameters is that the metallicity $([\mathrm{M} / \mathrm{H}]=-0.25 \mathrm{dex})$ is slightly subsolar, but the $\mathrm{C} / \mathrm{O}$ value is supersolar. Intriguingly, this situation is reminiscent of retrieval results for the T7.5pec dwarf SDSS $\mathrm{J} 1416+1348 \mathrm{~B}$, a companion to the sdL7 SDSS J1416+1348A (Line et al. 2017; Gonzales et al. 2020). Gonzales et al. (2020) found a slightly subsolar metallicity $([\mathrm{M} / \mathrm{H}] \approx-0.3 \mathrm{dex})$ for the SDSS J1416+1348AB system, but a roughly solar C/O ratio. With $T_{\text {eff }} \approx 600-700 \mathrm{~K}$ (Gonzales et al. 2020), SDSS $\mathrm{J} 1416+1348 \mathrm{~B}$ is significantly colder than WISEA $1553+6933$, so that it is not immediately evident whether the same spectral features are driving the elevated fits of $\mathrm{C} / \mathrm{O}$ relative to $[\mathrm{M} / \mathrm{H}]$ in both cases.

Taking into account the literature comparisons and model fits shown in Figures 4-6, we assign WISEA $1553+6933$ a spectral type of sdT4 \pm 0.5 . The subtype is quite well-constrained, given that the T3 and T5 standards in Figure 4 are very clearly worse matches to the WISEA $1553+6933$ spectrum than is the T4 standard. We adopt a metallicity of $\approx-0.5 \mathrm{dex}$ based on the bestfit models shown in Figures 5 and 6. In Table 2, we quote the 


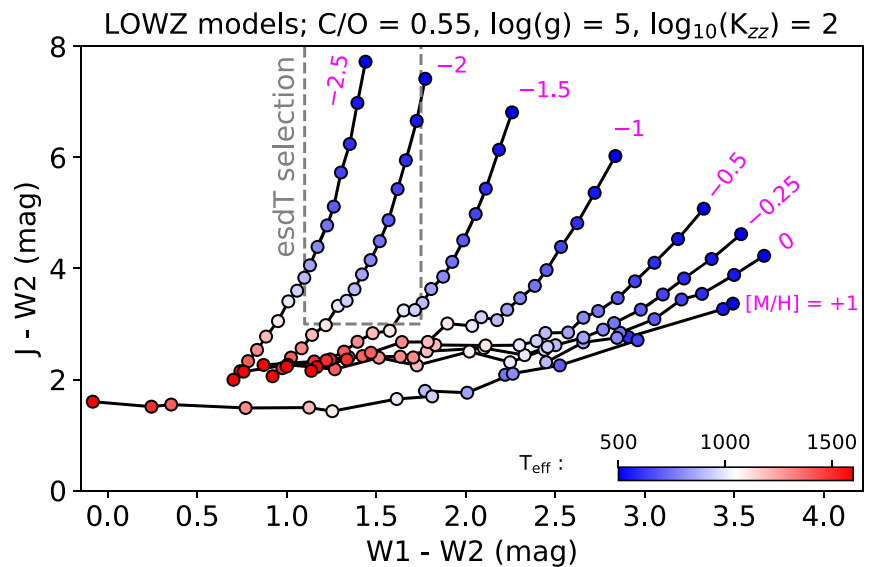

Figure 7. LOWZ model synthetic colors for "normal" brown dwarf parameters of $\mathrm{C} / \mathrm{O}=0.55, \log (g)=5.0$, and $\log _{10}\left(K_{\mathrm{zz}}\right)=2$. Each connected set of data points represents the temperature sequence $\left(500 \mathrm{~K} \leqslant T_{\text {eff }} \leqslant 1600 \mathrm{~K}\right)$ for one metallicity value. The metallicities range from supersolar $([\mathrm{M} / \mathrm{H}]=+1)$ to very metal-poor $([\mathrm{M} / \mathrm{H}]=-2.5)$. Magenta annotations list each curve's $[\mathrm{M} / \mathrm{H}]$ value. Each data point is color-coded by temperature, with blue being cold and red being relatively warm according to the inset color bar. The esdT candidate color-color selection box used in this work is shown as a dashed gray outline. As metallicity decreases from solar, the loci progressively compress and shift blueward in $\mathrm{W} 1-\mathrm{W} 2$ while expanding and shifting redward in $\mathrm{J}-\mathrm{W} 2$, leading the $[\mathrm{M} / \mathrm{H}] \lesssim-1.5$ curves to enter our esdT selection box.

WISEA $1553+6933$ effective temperature as $T_{\text {eff }}=1200 \pm 100$ $\mathrm{K}$ : the PHOENIX models favor temperatures of 1200-1300 K, and the LOWZ models favor a temperature of $1100 \mathrm{~K}$. The effective temperature from spectroscopic modeling is in good agreement with the photometrically estimated $T_{\text {eff }}=1076 \pm 89 \mathrm{~K}$ value from Section 4.2.

\section{LOWZ Model Synthetic Photometry}

Because the LOWZ models extend from blueward of $1 \mu \mathrm{m}$ all the way to $10 \mu \mathrm{m}$, we can generate synthetic near and midinfrared (MIR) colors corresponding to each model's set of physical parameters. Using MKO, WISE, and Spitzer transmission curves from the SVO Filter Profile Service (Rodrigo et al. 2012; Rodrigo \& Solano 2020), we computed each LOWZ model's J-W2, W1-W2, J-ch2, and ch1-ch2 colors in the Vega system. Figure 7 shows our LOWZ model synthetic colors in the same $\mathrm{J}-\mathrm{W} 2$ versus $\mathrm{W} 1-\mathrm{W} 2$ color-color space used to select this work's esdT candidates (see Figure 1).

Figure 7 includes synthetic colors for 136 LOWZ models, selected to have nominal "normal" brown dwarf parameters: $\mathrm{C} / \mathrm{O}=0.55, \log (g)=5$, and $\log _{10}\left(K_{\mathrm{zz}}\right)=2$. Each connected curve represents a unique $[\mathrm{M} / \mathrm{H}]$ value's temperature sequence, from $500 \mathrm{~K}$ to $1600 \mathrm{~K}$. The eight $[\mathrm{M} / \mathrm{H}]$ curves shown span from $[\mathrm{M} / \mathrm{H}]=+1$ to $[\mathrm{M} / \mathrm{H}]=-2.5$. The $[\mathrm{M} / \mathrm{H}]=0$ curve agrees reasonably well with the observed locus of "normal" $\mathrm{T}$ dwarfs shown as magenta points in Figure 1. A dramatic trend can be seen among the set of temperature sequence curves as metallicity becomes progressively lower. As metallicity decreases from $[\mathrm{M} / \mathrm{H}]=0$, the locus compresses and shifts blueward in terms of $\mathrm{W} 1-\mathrm{W} 2$, while expanding to push redward in $\mathrm{J}-\mathrm{W} 2$. This metallicity evolution of the T-dwarf sequence means that models with $[\mathrm{M} / \mathrm{H}] \lesssim-1.5$ enter our esdT candidate color-color selection box from Section 4, whereas the higher-metallicity models (including supersolar) shown do not occupy this area. Thus, our LOWZ models predict the strong $\mathbf{J}-\mathrm{W} 2$ color excess signature of the
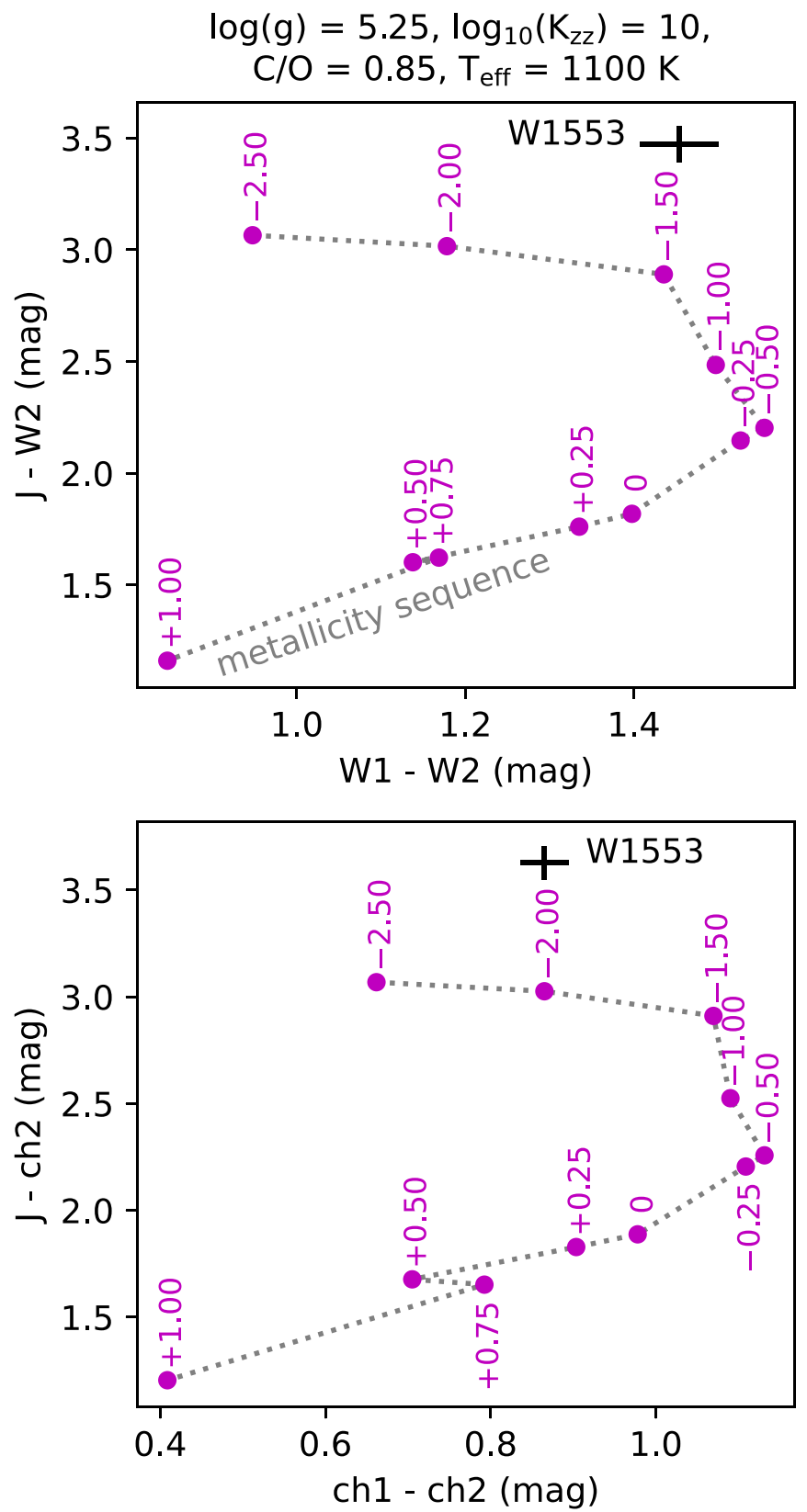

Figure 8. Synthetic colors of our LOWZ models as a function of metallicity, fixing all other physical parameters to those of the best-fit WISEA $1553+6933$ model from Section 5.2. Magenta annotations provide the $[M / H]$ value of each data point. WISEA $1553+6933$ is shown as a black plus mark in each panel. Top: WISE-based synthetic colors. Bottom: Spitzer-based synthetic colors. The WISE-based (Spitzer-based) synthetic broadband colors favor a very low photometric metallicity of $[\mathrm{M} / \mathrm{H}]=-1.5(-2)$ for WISEA $1553+6933$.

Schneider et al. (2020) esdTs. Our LOWZ model synthetic photometry also points to the possibility of finding very lowmetallicity $\mathrm{T}$ dwarfs with $\mathrm{J}-\mathrm{W} 2$ excesses even larger than observed for any of the subdwarfs in this work or Schneider et al. (2020), $5<\mathrm{J}-\mathrm{W} 2<8$. This motivates future brown dwarf searches and follow-up campaigns to seek examples of such extreme $\mathrm{J}-\mathrm{W} 2$ color at modest $\mathrm{W} 1-\mathrm{W} 2$ color in the $\sim 1$ -2 mag range.

Returning to the case study of WISEA $1553+6933$, we can also examine the metallicity sequence in this same synthetic color-color space while fixing all other physical parameters to be those of the best-fit LOWZ model from Figure 6. Figure 8 
shows the resulting metallicity sequence for $\log (g)=5.25$, $\log _{10}\left(K_{\mathrm{zz}}\right)=10, \mathrm{C} / \mathrm{O}=0.85$, and $T_{\text {eff }}=1100 \mathrm{~K}$. The top panel illustrates the trend of $\mathrm{J}-\mathrm{W} 2$ versus $\mathrm{W} 1-\mathrm{W} 2$, and the bottom panel shows the Spitzer-based analog that replaces W1 (W2) with $\operatorname{ch} 1$ (ch2). WISEA $1553+6933$ is closest to the $[\mathrm{M} /$ $\mathrm{H}]=-1.5(-2.0)$ model prediction in the WISE-based (Spitzer-based) color-color diagram. Thus, synthetic broadband colors of the LOWZ models yield a "photometric metallicity" of -1.5 to -2 for WISEA $1553+6933$, much lower than the best-fit $[\mathrm{M} / \mathrm{H}]=-0.25$ value derived from fitting the NIR spectral morphology (Section 5.2). In Table 2, we retain the relatively high metallicity value derived from our Section 5 fits, since we favor the spectroscopic analysis over broadband photometric estimates. WISEA 1553+6933 illustrates that, although the LOWZ models qualitatively capture observed trends of $\mathrm{J}-\mathrm{W} 2$ versus $\mathrm{W} 1-\mathrm{W} 2$, there is tension between the metallicities implied by NIR spectral morphology as compared to NIR-MIR broadband colors. JWST lowresolution spectroscopy of objects like WISEA 0414-5854, WISEA $1810-1010$ or WISEA $1553+6933$ covering $1-5 \mu \mathrm{m}$ with NIRSpec (Gardner et al. 2006; Bagnasco et al. 2007) would provide valuable insight into the detailed successes and mismatches of our LOWZ models in this mid-infrared wavelength range, helping to understand the key physical processes/parameters shaping the spectra and colors of $\mathrm{T}$ subdwarfs.

\section{Conclusion}

We have presented discoveries and follow-up of three T-type subdwarf candidates identified by the Backyard Worlds: Planet 9 citizen science project. These objects have large total proper motions in the range of 0 ". $9-2$ !" $2 \mathrm{yr}^{-1}$, with correspondingly high tangential velocity estimates $\left(\sim 180-400 \mathrm{~km} \mathrm{~s}^{-1}\right)$ indicative of membership in the Milky Way thick disk or halo. Their photometry places them in the same unusual region of colorcolor space as the first two known examples of the esdT spectral class (Schneider et al. 2020). We have also published an extensive new grid of low-temperature, low-metallicity model atmospheres. Going forward, these models will aid in the characterization/ classification of the $\mathrm{T}$ subdwarf population.

Keck/NIRES spectroscopy indicates that WISEA 1553+6933 is a T4 subdwarf with an effective temperature of $T_{\text {eff }}=1200 \pm$ $100 \mathrm{~K}$ and metallicity $\approx-0.5$ dex. Although WISEA $1553+6933$ has the largest W2 reduced proper motion among all known L- and T-type subdwarfs, suggesting that it may have unusually high kinematics and/or low luminosity even relative to other $\mathrm{T}$ subdwarfs, its near-infrared spectral morphology does not indicate a metallicity sufficiently low to be classified as an esdT. On the other hand, comparison of the WISEA $1553+6933$ broadband photometry from $\sim 1-5 \mu \mathrm{m}$ against the LOWZ models favors a lower metallicity $\lesssim-1.5$ dex.

Our understanding of all three objects presented in this work would benefit from additional astrometry, in order to obtain trigonometric parallaxes. Trigonometric parallaxes would specify how subluminous these subdwarfs are relative to "normal" T dwarfs. Determining luminosities of the two known esdTs and other potentially similar objects may help to better map/define the sdT/esdT sequence.

Additional near-infrared photometry of this study's sample is also needed. In particular, a J-band detection of CWISE 0738 -6643 would allow us to pinpoint its location in color-color space. Deeper H- and K-band follow-up imaging is needed for all three members of the present sample, and would allow us to place these objects within additional color-color diagrams (e.g., Figure 3 of Schneider et al. 2020).

Finally, near-infrared spectroscopy is needed for the two new discoveries presented in this work, CWISE 0738-6643 and CWISE 2217-1454, to gauge their metallicities and temperatures, thereby determining whether either is a new member of the esdT spectral class.

Backyard Worlds will continue mining the WISE/NEOWISE data set for faint moving objects in search of more surprising substellar discoveries with anomalous properties.

The Backyard Worlds: Planet 9 team would like to thank the many Zooniverse volunteers who have participated in this project, from providing feedback during the beta review stage to classifying flipbooks to contributing to the discussions on TALK. We would also like to thank the Zooniverse web development team for their work creating and maintaining the Zooniverse platform and the Project Builder tools. This research was supported by NASA grant 2017-ADAP17-0067. This material is based upon work supported by the National Science Foundation under grant No. 2007068, 2009136, and 2009177. F.M. acknowledges support from grant 80NSSC20K0452 under the NASA Astrophysics Data Analysis Program. S.L.C. acknowledges the support of a STFC Ernest Rutherford Fellowship. The CatWISE effort is led by the Jet Propulsion Laboratory, California Institute of Technology, with funding from NASA's Astrophysics Data Analysis Program. Support for this work was provided by NASA through the NASA Hubble Fellowship grant HST-HF251447.001-A awarded by the Space Telescope Science Institute, which is operated by the Association of Universities for Research in Astronomy, Inc., for NASA, under contract NAS5-26555. The work of P.R.M.E. was carried out at the Jet Propulsion Laboratory, California Institute of Technology, under a contract with NASA. This publication makes use of data products from the Wide-field Infrared Survey Explorer, which is a joint project of the University of California, Los Angeles, and the Jet Propulsion Laboratory/California Institute of Technology, funded by the National Aeronautics and Space Administration. The UHS is a partnership between the UK STFC, The University of Hawaii, The University of Arizona, Lockheed Martin and NASA. The VISTA Data Flow System pipeline processing and science archive are described in Irwin et al. (2004), Hambly et al. (2008), and Cross et al. (2012). We have used data from VHS DR6. This research has made use of the NASA/IPAC Infrared Science Archive, which is funded by the National Aeronautics and Space Administration and operated by the California Institute of Technology. Some of the data presented herein were obtained at the W. M. Keck Observatory, which is operated as a scientific partnership among the California Institute of Technology, the University of California and the National Aeronautics and Space Administration. The Observatory was made possible by the generous financial support of the W. M. Keck Foundation. The authors wish to recognize and acknowledge the very significant cultural role and reverence that the summit of Maunakea has always had within the indigenous Hawaiian community. We are most fortunate to have the opportunity to conduct observations from this mountain. Based on observations obtained at the international Gemini Observatory, a program of NSF's NOIRLab, which is managed by the Association of Universities for Research in Astronomy (AURA) under a cooperative agreement with the National Science Foundation on behalf of the Gemini Observatory 
partnership: the National Science Foundation (United States), National Research Council (Canada), Agencia Nacional de Investigacion y Desarrollo (Chile), Ministerio de Ciencia, Tecnologia e Innovacion (Argentina), Ministerio da Ciencia, Tecnologia, Inovacoes e Comunicacoes (Brazil), and Korea Astronomy and Space Science Institute (Republic of Korea). This research has made use of the VizieR catalog access tool, CDS, Strasbourg, France (DOI: $10.26093 / \mathrm{cds} /$ vizier). The original description of the VizieR service was published in Ochsenbein et al. (2000). This research has made use of the SVO Filter Profile Service (http://svo2.cab.inta-csic.es/theory/fps/) supported from the Spanish MINECO through grant AYA201784089.

Facilities: Keck(NIRES, MOSFIRE), WISE, Spitzer (IRAC), Gemini North(NIRI).

Software: WiseView (Caselden et al. 2018), IRAF (Tody 1986), SPLAT (Burgasser \& Splat Development Team 2017).

\section{ORCID iDs}

Aaron M. Meisner (ib https://orcid.org/0000-0002-1125-7384 Adam C. Schneider (i) https://orcid.org/0000-0002-6294-5937 Adam J. Burgasser (i) https://orcid.org/0000-0002-6523-9536 Federico Marocco (iD https://orcid.org/0000-0001-7519-1700 Michael R. Line (iD https://orcid.org/0000-0002-2338-476X Jacqueline K. Faherty (1) https://orcid.org/0000-00016251-0573

J. Davy Kirkpatrick (iD https://orcid.org/0000-00034269-260X

Dan Caselden (iD https://orcid.org/0000-0001-7896-5791

Marc J. Kuchner (iD https://orcid.org/0000-0002-2387-5489

Jonathan Gagné (i) https://orcid.org/0000-0002-2592-9612

Christopher Theissen (iD https://orcid.org/0000-0002-

9807-5435

Roman Gerasimov (ib https://orcid.org/0000-0003-0398-639X Christian Aganze (1) https://orcid.org/0000-0003-2094-9128 Chih-chun Hsu (ib https://orcid.org/0000-0002-5370-7494

John P. Wisniewski (i) https://orcid.org/0000-0001-9209-1808 Sarah L. Casewell (iD https://orcid.org/0000-0003-2478-0120 Daniella C. Bardalez Gagliuffi (1) https://orcid.org/0000-00018170-7072

Sarah E. Logsdon (iD https://orcid.org/0000-0002-9632-9382

Katelyn Allers (i) https://orcid.org/0000-0003-0580-7244

John H. Debes (D) https://orcid.org/0000-0002-1783-8817

Nikolaj Stevnbak Andersen (1) https://orcid.org/0000-00034714-3829

Sam Goodman (iD https://orcid.org/0000-0003-2236-2320

Léopold Gramaize (iD https://orcid.org/0000-0002-8960-4964

Arttu Sainio (ib https://orcid.org/0000-0003-4864-5484

Michael C. Cushing (i) https://orcid.org/0000-0001-7780-3352

\section{References}

Alavi, S. F., \& Shayesteh, A. 2018, MNRAS, 474, 2

Allard, F., Homeier, D., Freytag, B., et al. 2013, MSAIS, 24, 128

Allard, N. F., Spiegelman, F., \& Kielkopf, J. F. 2007, A\&A, 465, 1085

Allard, N. F., Spiegelman, F., Leininger, T., \& Molliere, P. 2019, A\&A, 628, A120

Amundsen, D. S., Tremblin, P., Manners, J., Baraffe, I., \& Mayne, N. J. 2017, A\&A, 598, A97

Arcangeli, J., Désert, J.-M., Line, M. R., et al. 2018, ApJL, 855, L30

Artigau, E., Doyon, R., Vallee, P., Riopel, M., \& Nadeau, D. 2004, Proc. SPIE, 5492,1479

Bagnasco, G., Kolm, M., Ferruit, P., et al. 2007, Proc. SPIE, 6692, 66920M
Bardalez Gagliuffi, D. C., Faherty, J. K., Schneider, A. C., et al. 2020, ApJ, 895,145

Barton, E. J., Yurchenko, S. N., \& Tennyson, J. 2013, MNRAS, 434, 1469

Baxter, C., Désert, J.-M., Parmentier, V., et al. 2020, A\&A, 639, A36

Beatty, T. G., Wong, I., Fetherolf, T., et al. 2020, AJ, 160, 211

Burgasser, A. J. 2014, in ASI Conf. Ser. 11, Int. Workshop on Stellar Spectral Libraries 2014 (Bengaluru: ASI), 7

Burgasser, A. J. \& Splat Development Team 2017, in ASI Conf. Ser. 14, Int. Workshop on Spectral Stellar Libraries 2017 (Bengaluru: ASI), 7

Burgasser, A. J., Geballe, T. R., Leggett, S. K., Kirkpatrick, J. D., \& Golimowski, D. A. 2006, ApJ, 637, 1067

Burgasser, A. J., Kirkpatrick, J. D., \& Lépine, S. 2005, in Proc. of the 13th Cambridge Workshop on Cool Stars, Stellar Systems and the Sun, 560, ed. F. Favata, G. A. Hussain, \& B. Battrick (Paris: ESA), 237, arXiv:astro-ph/ 0409178

Burgasser, A. J., McElwain, M. W., Kirkpatrick, J. D., et al. 2004, AJ, 127,2856

Burningham, B., Smith, L., Cardoso, C. V., et al. 2014, MNRAS, 440, 359

Burrows, A. 2005, Natur, 433, 261

Casali, M., Adamson, A., Alves de Oliveira, C., et al. 2007, A\&A, 467, 777

Caselden, D., Westin, P., III, Meisner, A., Kuchner, M., \& Colin, G. 2018, WiseView: Visualizing Motion and Variability of Faint WISE Sources, Astrophysics Source Code Library, ascl:1806.004

Colón, K. D., Kreidberg, L., Welbanks, L., et al. 2020, AJ, 160, 280

Cross, N. J. G., Collins, R. S., Mann, R. G., et al. 2012, A\&A, 548, A119

Cushing, M. C., Vacca, W. D., \& Rayner, J. T. 2004, PASP, 116, 362

Cutri, R. M., Wright, E. L., Conrow, T., et al. 2013, Explanatory Supplement to the AllWISE Data Release Products, Tech. rep.

Debes, J. H., Thévenot, M., Kuchner, M. J., et al. 2019, ApJL, 872, L25

Dey, A., Rabinowitz, D., Karcher, A., et al. 2016, Proc. SPIE, 9908, 99082C

Dey, A., Schlegel, D. J., Lang, D., et al. 2019, AJ, 157, 168

Dye, S., Lawrence, A., Read, M. A., et al. 2018, MNRAS, 473, 5113

Eisenhardt, P. R. M., Marocco, F., Fowler, J. W., et al. 2020, ApJS, 247, 69

Faherty, J. K., Goodman, S., Caselden, D., et al. 2020, ApJ, 889, 176

Fitzpatrick, M., Olsen, K., Eychaner, G., et al. 2019, in ASP Conf. Ser. 523, The NOAO Data Lab: Design, Capabilities, and Community Development, ed. P. J. Teuben, M. W. Pound, B. A. Thomas, \& E. M. Warner (San Francisco, CA: ASP), 233

Gaia Collaboration, Brown, A. G. A., Vallenari, A., et al. 2018, A\&A, 616, A1 Gardner, J. P., Mather, J. C., Clampin, M., et al. 2006, SSRv, 123, 485

Gerasimov, R., Homeier, D., Burgasser, A., \& Bedin, L. R. 2020, RNAAS, 4,214

Gharib-Nezhad, E., \& Line, M. R. 2019, ApJ, 872, 27

GharibNezhad, E., Shayesteh, A., \& Bernath, P. F. 2013, MNRAS, 432, 2043 Gonzales, E. C., Burningham, B., Faherty, J. K., et al. 2020, ApJ, 905, 46

Gonzales, E. C., Faherty, J. K., Gagné, J., Artigau, É, \& Bardalez Gagliuffi, D. 2018, ApJ, 864, 100

Greco, J. J., Schneider, A. C., Cushing, M. C., Kirkpatrick, J. D., \& Burgasser, A. J. 2019, AJ, 158, 182

Griffith, R. L., Kirkpatrick, J. D., Eisenhardt, P. R. M., et al. 2012, AJ, 144, 148 Hambly, N. C., Collins, R. S., Cross, N. J. G., et al. 2008, MNRAS, 384, 637 Harris, G. J., Larner, F. C., Tennyson, J., et al. 2008, MNRAS, 390, 143 Hauschildt, P. H., \& Baron, E. 1999, JCoAM, 109, 41

Hewett, P. C., Warren, S. J., Leggett, S. K., \& Hodgkin, S. T. 2006, MNRAS, 367,454

Hodapp, K. W., Jensen, J. B., Irwin, E. M., et al. 2003, PASP, 115, 1388 Irwin, M. J. 2008, in ESO Astrophysics Symp., 2007 ESO Instrument Calibration Workshop, ed. A. Kaufer \& F. Kerber (Berlin: Springer), 541 Irwin, M. J., Lewis, J., Hodgkin, S., et al. 2004, Proc. SPIE, 5493, 411 Jalowiczor, P. A., Casewell, S., Schneider, A. C., et al. 2021, RNAAS, 5, 76 John, T. L. 1988, A\&A, 193, 189

Kirkpatrick, J. D., Cushing, M. C., Gelino, C. R., et al. 2011, ApJS, 197, 19 Kirkpatrick, J. D., Gelino, C. R., Faherty, J. K., et al. 2021, ApJS, 253, 7 Kirkpatrick, J. D., Martin, E. C., Smart, R. L., et al. 2019b, ApJS, 240, 19 Kirkpatrick, J. D., Metchev, S. A., Hillenbrand, L. A., et al. 2019a, BAAS, 51, 108

Kuchner, M. J., Faherty, J. K., Schneider, A. C., et al. 2017, ApJL, 841, L19 Kurucz, R. L. 1993, SYNTHE Spectrum Synthesis Programs and Line Data (Cambridge, MA: Smithsonian Astrophysical Observatory)

Lang, D. 2014, AJ, 147, 108

Lawrence, A., Warren, S. J., Almaini, O., et al. 2007, MNRAS, 379, 1599

Leggett, S., Apai, D., Burgasser, A., et al. 2019, BAAS, 51, 95

Line, M. R., Marley, M. S., Liu, M. C., et al. 2017, ApJ, 848, 83

Lodders, K., \& Palme, H. 2009, M\&PSA, 72, 5154

Luhman, K. L. 2014, ApJ, 786, L18

Luyten, W. J. 1922, LicOB, 336, 135 
Mace, G. N., Kirkpatrick, J. D., Cushing, M. C., et al. 2013a, ApJS, 205, 6 Mace, G. N., Kirkpatrick, J. D., Cushing, M. C., et al. 2013b, ApJ, 777, 36 Mansfield, M., Bean, J. L., Line, M. R., et al. 2018, AJ, 156, 10

Marley, M. S., \& Robinson, T. D. 2015, ARA\&A, 53, 279

Marocco, F., Caselden, D., Meisner, A. M., et al. 2019, ApJ, 881, 17

Marocco, F., Eisenhardt, P. R. M., Fowler, J. W., et al. 2021, ApJS, 253, 8

McKay, C. P., Pollack, J. B., \& Courtin, R. 1989, Icar, 80, 23

McKemmish, L. K., Masseron, T., Hoeijmakers, H. J., et al. 2019, MNRAS, 488, 2836

McKemmish, L. K., Yurchenko, S. N., \& Tennyson, J. 2016, MNRAS, 463, 771

McLean, I. S., Steidel, C. C., Epps, H. W., et al. 2012, Proc. SPIE, 8446, 84460J

McMahon, R. G., Banerji, M., Gonzalez, E., et al. 2013, Msngr, 154, 35

Meisner, A. M., Faherty, J. K., Kirkpatrick, J. D., et al. 2020, ApJ, 899, 123

Meisner, A. M., Lang, D., \& Schlegel, D. J. 2018, AJ, 156, 69

Nakajima, T., Oppenheimer, B. R., Kulkarni, S. R., et al. 1995, Natur, 378, 463

Nidever, D. L., Dey, A., Olsen, K., et al. 2018, AJ, 156, 131

Ochsenbein, F., Bauer, P., \& Marcout, J. 2000, A\&AS, 143, 23

Pinfield, D. J., Gomes, J., Day-Jones, A. C., et al. 2014, MNRAS, 437, 1009

Piskorz, D., Buzard, C., Line, M. R., et al. 2018, AJ, 156, 133

Polyansky, O. L., Kyuberis, A. A., Zobov, N. F., et al. 2018, MNRAS, 480, 2597

Racine, R. 1978, JRASC, 72, 324

Reid, I. N., \& Metchev, S. A. 2008, in The Brown Dwarf-Exoplanet Connection, ed. J. W. Mason (Chichester: Praxis), 115

Richard, C., Gordon, I. E., Rothman, L. S., et al. 2012, JQSRT, 113, 1276

Rodrigo, C., \& Solano, E. 2020, in Contributions to the XIV.0 Scientific Meeting (virtual) of the Spanish Astronomical Society (Barcelona: SEA), 182

Rodrigo, C., Solano, E., \& Bayo, A. 2012, SVO Filter Profile Service Version 1.0, IVOA Working Draft 15 October 2012, 2012, doi:10.5479/ADS/bib/ 2012ivoa.rept.1015R

Rothermich, A., Schneider, A. C., Faherty, J. K., et al. 2021, RNAAS, 5, 18
Rothman, L. S., Gordon, I. E., Babikov, Y., et al. 2013, JQSRT, 130, 4

Rothman, L. S., Gordon, I. E., Barber, R. J., et al. 2010, JQSRT, 111, 2139

Saumon, D., Marley, M. S., Abel, M., Frommhold, L., \& Freedman, R. S. 2012, ApJ, 750, 74

Schlafly, E. F., Green, G. M., Lang, D., et al. 2018, ApJS, 234, 39

Schlafly, E. F., Meisner, A. M., \& Green, G. M. 2019, ApJS, 240, 30

Schneider, A. C., Burgasser, A. J., Gerasimov, R., et al. 2020, ApJ, 898, 77

Schneider, A. C., Greco, J., Cushing, M. C., et al. 2016, ApJ, 817, 112

Simpson, R., Page, K. R., \& De Roure, D. 2014, in Proc. of the 23rd Int. Conf. on World Wide Web, WWW '14 Companion (New York: Association for Computing Machinery), 1049

Skrutskie, M. F., Cutri, R. M., Stiening, R., et al. 2006, AJ, 131, 1163

Sousa-Silva, C., Al-Refaie, A. F., Tennyson, J., \& Yurchenko, S. N. 2015 MNRAS, 446, 2337

Tennyson, J., \& Yurchenko, S. N. 2012, MNRAS, 425, 21

Tody, D. 1986, Proc. SPIE, 627, 733

Toon, O. B., McKay, C. P., Ackerman, T. P., \& Santhanam, K. 1989, JGR, 94 16287

Vacca, W. D., Cushing, M. C., \& Rayner, J. T. 2003, PASP, 115, 389

Wende, S., Reiners, A., Seifahrt, A., \& Bernath, P. F. 2010, A\&A, 523, A58

Wilson, J. C., Henderson, C. P., Herter, T. L., et al. 2004, Proc. SPIE, 5492, 1295

Wright, E., Eisenhardt, P., Mainzer, A., et al. 2010, AJ, 140, 1868

Yadin, B., Veness, T., Conti, P., et al. 2012, MNRAS, 425, 34

Yurchenko, S. N., Barber, R. J., \& Tennyson, J. 2011, MNRAS, 413, 1828

Yurchenko, S. N., \& Tennyson, J. 2014, MNRAS, 440, 1649

Yurchenko, S. N., Williams, H., Leyland, P. C., Lodi, L., \& Tennyson, J. 2018, MNRAS, 479, 1401

Zalesky, J. A., Line, M. R., Schneider, A. C., \& Patience, J. 2019, ApJ, 877,24

Zhang, Z. H., Burgasser, A. J., Gálvez-Ortiz, M. C., et al. 2019, MNRAS, 486,1260

Zhang, Z. H., Galvez-Ortiz, M. C., Pinfield, D. J., et al. 2018, MNRAS, 480,5447 